\title{
Open boundary particle-in-cell simulation of dipolarization front propagation
}

\author{
Alex Klimas, ${ }^{1,2}$ Kyoung-Joo Hwang, ${ }^{1}$ Adolfo F. -Viñas, ${ }^{3}$ and Melvyn L. Goldstein ${ }^{3}$ \\ Received 2 August 2013; revised 6 November 2013; accepted 9 December 2013.
}

[1] First results are presented from an ongoing open boundary $2 \frac{1}{2} \mathrm{D}$ particle-in-cell simulation study of dipolarization front (DF) propagation in Earth's magnetotail. At this stage, this study is focused on the compression, or pileup, region preceding the DF current sheet. We find that the earthward acceleration of the plasma in this region is in general agreement with a recent DF force balance model. A gyrophase bunched reflected ion population at the leading edge of the pileup region is reflected by a normal electric field in the pileup region itself, rather than through an interaction with the current sheet. We discuss plasma wave activity at the leading edge of the pileup region that may be driven by gradients, or by reflected ions, or both; the mode has not been identified. The waves oscillate near but above the ion cyclotron frequency with wavelength several ion inertial lengths. We show that the waves oscillate primarily in the perpendicular magnetic field components, do not propagate along the background magnetic field, are right handed elliptically (close to circularly) polarized, exist in a region of high electron and ion beta, and are stationary in the plasma frame moving earthward. We discuss the possibility that the waves are present in plasma sheet data, but have not, thus far, been discovered.

Citation: Klimas, A., K.-J. Hwang, A. F. -Viñas, and M. L. Goldstein (2014), Open boundary particle-in-cell simulation of dipolarization front propagation, J. Geophys. Res. Space Physics, 119, doi:10.1002/2013JA019282.

\section{Introduction}

[2] It is generally accepted that a dipolarization front (DF) in Earth's magnetotail is a north-south oriented, thin dawn to dusk directed current sheet with associated disturbances that can propagate large distances, usually earthward, as a recognizable coherent structure [Runov et al., 2009; Sergeev et al., 2009; Runov et al., 2011a; Sergeev et al., 2012]. The current sheet, whose thickness remains of the order of a thermal proton gyroradius [Hwang et al., 2011; Schmid et al., 2011], separates two magnetic domains with a significantly stronger northward directed magnetic field, $B_{z}$, on its trailing side and, often, a small dip in this field component just preceding it. Thus, an identifying characteristic of a DF in spacecraft observations is the resulting asymmetric bipolar structure in the $B_{z}$ time series with the following increase in the field strength appearing almost step-like. In the magnetotail, DFs are often observed at the leading edges of fast earthward flows and flow bursts within bursty bulk flow events [Angelopoulos et al., 1994; Runov et al., 2009; Hwang et al., 2011; Runov et al., 2011a, 2011b]. As such, DFs are thought to separate

\footnotetext{
${ }^{1} \mathrm{GPHI}$ at University of Maryland, Baltimore County, Baltimore, Maryland, USA. USA.

${ }^{2}$ Emeritus at NASA Goddard Space Flight Center, Greenbelt, Maryland,

${ }^{3}$ NASA Goddard Space Flight Center, Greenbelt, Maryland, USA.

Corresponding author: A. Klimas, Emeritus at NASA Goddard Space Flight Center, Greenbelt, MD 20771, USA. (alex.klimas@nasa.gov)

(C2013. American Geophysical Union. All Rights Reserved. 2169-9380/14/10.1002/2013JA019282
}

the cold, dense, ambient plasma sheet population from the hot, tenuous outflow of reconnection activity deeper in the tail. It has been noted that DFs propagate at approximately the local Alfvén speed, which is consistent with this scenario [Hwang et al., 2011]. Further, particle-in-cell (PIC) simulations of impulsive reconnection events have supported this interpretation [Sitnov et al., 2009; Sitnov and Swisdak, 2011]. The fast flows slow rapidly as they encounter Earth's dominant dipolar magnetic field and a strong pressure gradient [Shiokawa et al., 1997; Dubyagin et al., 2010; Xing et al., 2010] in the transition region between the dipole-like inner and tail-like outer plasma sheet. It is thought that substorm or substorm-like auroral breakup activity is triggered in the inner transition region by this braking mechanism [Ohtani, 2004; Nishimura et al., 2010; Mende et al., 2011; Sergeev et al., 2012]. Thus, the flows provide a link between reconnection activity deeper in the tail with consequent substorm and substorm-like auroral activity. The flow-burst braking mechanism involved in this coupling in the inner magnetosphere is not well understood. This mechanism and the role of the DF in plasma acceleration and injection into the inner magnetosphere are subjects under investigation at present[Nakamura et al., 2009; Ohtani et al., 2009; Sergeev et al., 2009; Dubyagin et al., 2010; Panov et al., 2010a, 2010b; Xing et al., 2010; Hwang et al., 2011; Sergeev et al., 2012].

[3] With the arrival of a DF at the position of a spacecraft in the plasma sheet, it has been found that the appearance of the earthward flow does not coincide exactly with that of the current sheet [Sergeev et al., 2009; Runov et al., 2011b, 2011a; Sergeev et al., 2012]. The current sheet is often embedded within the leading portion of the fast flow with a 
precursor in front of it. This leading "compression" or "pileup" region typically lasts approximately one minute during which the flow speed and plasma pressure and density rise slowly before the arrival of the current sheet. The pileup region is clearly driven forward by the approaching current sheet; it appears to consist of a mixture of upstream plasma plus plasma that has been reflected forward by the impending DF proper. There are two available explanations for the reflection mechanism: one based on MHD force balance [Li et al., 2011] and the second based on a kinetic model of individual particle reflection at the current sheet [Zhou et al., 2010, 2011, 2012a, 2012b].

[4] Li et al. [2011] have studied a THEMIS [Angelopoulos et al., 2008; Sibeck and Angelopoulos, 2008] DF event observed in the magnetotail at $\mathrm{X} \simeq-10 R_{E}$ by the closely separated $\mathrm{P} 3 / \mathrm{THD}, \mathrm{P} 4 / \mathrm{THE}$, and $\mathrm{P} 5 / \mathrm{THA}$ probes, and at $\mathrm{X} \simeq-18 R_{E}$ by the $\mathrm{P} 2 / \mathrm{THC}$ probe. Based on the time delay between the inner and outer observations, the DF traveled earthward at better than $500 \mathrm{~km} / \mathrm{s}$. The DF was embedded within a fast earthward directed bulk flow. Starting approximately one minute before the arrival of the current sheet, the flow speed slowly increased at the inner probes, consistent with the results of Runov et al. [2011b, 2011a]. Using the fortuitous placement of the inner probes, $\mathrm{Li}$ et al. were able to evaluate the magnetic curvature force density and the total pressure gradient force density acting on the plasma in the neighborhood of the current sheet. They found that despite the increased radius of curvature following the current sheet, the earthward directed curvature force density increased due to the magnetic field increase within the dipolarized flow burst. Since, in this following region, the pressure gradient force remained unchanged compared to quiet conditions, the magnetic field curvature force was found responsible for accelerating the plasma in the bulk flow region following the current sheet. In the leading pileup region, $\mathrm{Li}$ et al. found the normally tailward directed pressure gradient force reduced compared to quiet conditions while the curvature force density remained consistent with quiet conditions. Thus, they concluded that the pressure gradient force was responsible for the formation of the leading pileup region and for the earthward acceleration of its ambient plasma. Li et al. suggested that flow bursts accompanied by a large amplitude DF will penetrate deeper into the dipole field dominated near-Earth plasma sheet, implicating the DF in the flow burst braking and auroral breakup triggering process. Zhou et al. [2010, 2011] had earlier shown that the formation of the compression region could be explained as an ion pickup region populated by particles reflected at the current sheet. $\mathrm{Li}$ et al. suggested that a distribution function analysis could tell which of the two mechanisms was responsible for the appearance of the region.

[5] In a series of recent papers, Zhou et al. [2010, 2011, $2012 a, 2012 b]$ have investigated the relationship between fast flows, DFs, associated pileup regions, and the emergence of field aligned particle beams in the plasma sheet boundary layer (PSBL). Their results follow from their case studies and statistical surveys of THEMIS 2008 and 2009 tail season observations plus supporting test particle simulations. In its latest version, the simulation model is based on a realistic two-dimensional kinetic plasma sheet model, a slight modification of the Pritchett and Buchner [1995] model, containing a monotonically decreasing neutral sheet $B_{z}$ with increasing depth into the tail. On top of this background, an electromagnetic model of a propagating DF is imposed. This consists of a $B_{z}$ field with a finite width in the dawn-dusk direction and containing a sharp step-like increase at a position that propagates earthward at a fixed speed. A dawn-dusk inductive electric field is also included to satisfy Faraday's Law for the varying magnetic field. The propagating DF model has no particle content. At each step in the simulation, taking the presence of the DF component into account, particles are integrated backward in time from their position at a virtual spacecraft to their origin in the background plasma sheet model. Liouville's theorem is invoked to relate the particle distribution at the spacecraft to that at the origin in the kinetic background model. The result is the formation of an energetic particle precursor to an arriving current sheet that is populated by particles that have been reflected and accelerated at the current sheet. The model does reproduce observed upstream earthward and dawnward drifts [Zhou et al., 2012b]. Further, in this model, particles in the central plasma sheet arrive 10's of seconds early while particles in the PSBL arrive minutes early, in very good agreement with THEMIS observations [Zhou et al., 2012a]. From this point of view, the pileup region is populated by particles reflected and energized by the magnetic and electric fields of the traveling current sheet. The particles in the central plasma sheet are limited to a region earthward of the current sheet of extent approximately one thermal gyro radius in the $B_{z}$ field while particles in the PSBL move more freely earthward.

[6] In this paper, we report first results from an ongoing effort to develop a particle-in-cell (PIC) simulation model that will be analogous to the Zhou et al. [2012a] model, but fully self-consistent. Our intent is to use newly available open boundary techniques [Klimas et al., 2008, 2010, 2012] to drive a DF across a simulation domain that initially represents a quiet plasma sheet. Initial results that we present below demonstrate, first, that the open boundary, driven simulation approach is feasible. Further, we find that many of the observed DF properties are reproduced by this model. A pileup region is produced whose properties are generally consistent with the force balance picture [Li et al., 2011] but differ from the particle pick up picture [Zhou et al., 2010, 2011, 2012a, 2012b]. The simulated pileup region is, indeed, populated by gyrophase bunched reflected ions. However, these ions are reflected through an interaction with the pileup region itself, not through an interaction with the current sheet. Such a self-consistent interaction possibility is not included in the Zhou et al. particle tracing model. The present version of our model is highly idealized. It will be important to verify these preliminary results using more sophisticated versions of the driven DF PIC simulation approach.

[7] We find that wave activity at the leading edge of the pileup region is a persistent feature of our DF simulations. Wave activity growth coincides with the maximum gradient in density, pressure, field strength, etc., at the leading edge. We show that this is also the region where the ion velocity distribution is highly nongyrotropic due to the presence of reflected ions. Both the steep gradients and the nongyrotropic velocity distributions are candidates for exciting the waves. However, we have not identified the wave mode(s) involved. The possibility that the waves are present in plasma sheet data but have not, thus far, been discovered is discussed. 


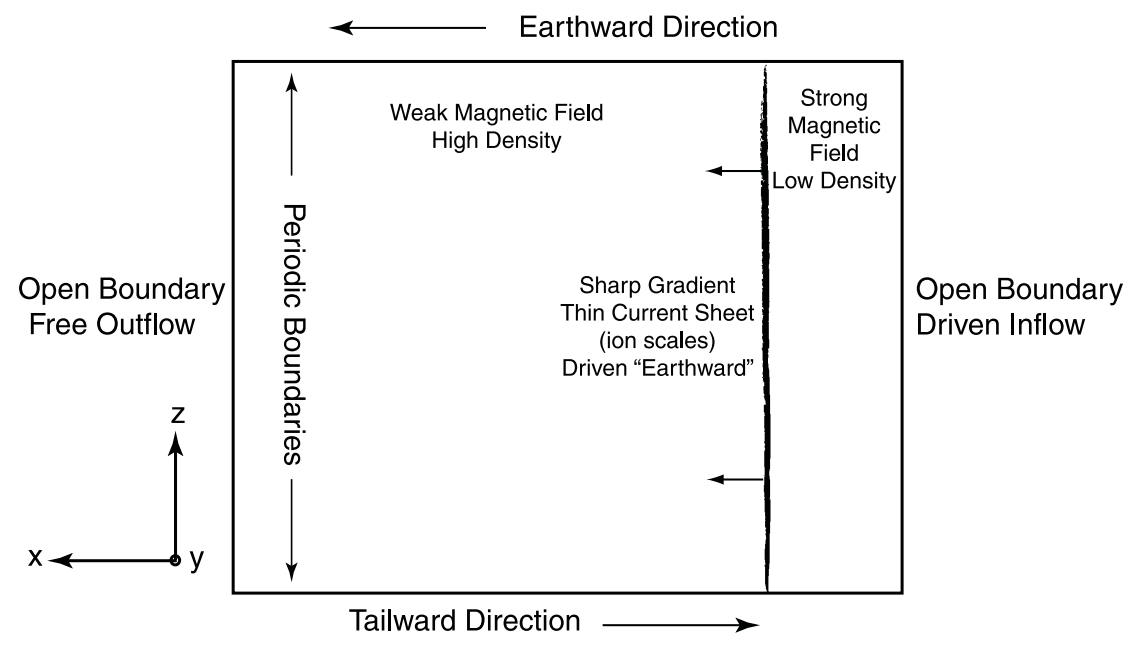

Figure 1. Driven DF simulation setup.

\section{Simulation Setup}

[8] Figure 1 gives an overview of the simulation setup for the driven DF model. The simulation models the passage of a propagating DF through a portion of the central plasma sheet in Earth's magnetotail. The earthward direction is to the left, in the direction of increasing $x$, with the deeper tail to the right. The $z$ axis points to the north and the $y$ axis, from dawn to dusk. Plasma is driven into the simulation domain through the open right-side boundary through the application of an out-of-plane electric field, $E_{y}$, on the boundary and plasma is free to pass out of the simulation domain through the open left-side boundary. The initial magnetic field is solely in the $z$ direction. In this version of the model, the upper and lower boundary conditions are periodic. This, plus the lack of an $x$ component in the initial magnetic field, produces the most serious limitations in the applicability of this idealized model. Work on a more generalized model in which these restrictions are removed is in progress. It will be very important to verify that the conclusions that we present in this paper remain valid.

[9] The simulation is initialized with the magnetic field in the $z$ direction (northward) given by [Pritchett, 2008],

$$
B_{z}=B_{0}\left(R-\tanh \left(\frac{x}{\lambda}\right)\right)
$$

with a current sheet in the $y$ direction (dawn-dusk) of thickness $\lambda$ in the $x$ direction given by

$$
j_{y}=\frac{B_{0}}{\lambda} \cosh ^{-2}\left(\frac{x}{\lambda}\right)
$$

[10] The parameter $B_{0}$ measures the initial overall strength of the DF, $\lambda$ can be adjusted to control the initial thickness of the $\mathrm{DF}$, and $R$ is a free parameter that can be used to control the relative strengths of the field on either side of the current sheet. Notice that

$$
\begin{aligned}
& B_{z} \underset{x / \lambda \ll 0}{\sim} B_{0}(R+1) \\
& B_{z} \underset{x / \tilde{\lambda \gg 0}}{ } B_{0}(R-1)
\end{aligned}
$$

[11] Standard symmetric reconnection simulations use $R=0$, asymmetric simulations (e.g., Pritchett [2008]) are based on $0<R<1$, and for this case we choose $R>1$, with the initial ratio of the field strengths on either side of the DF controlled by our choice.

[12] To achieve an equilibrium initial state, we balance the magnetic field pressure with the plasma pressure so that $P(x)+$ $B_{z}^{2} / 8 \pi=$ const $=C$. We assume Gaussian particle distributions in this initial state and further assume that $P(x)=n(x) k$ $\left(T_{i}+T_{e}\right)$, in which $n(x)$ is the ion or electron density and the ion and electron temperatures are uniform in $x$. We make the further assumption that $B_{0}^{2} / 8 \pi=\alpha n_{0} k\left(T_{i}+T_{e}\right)$ in which $\alpha$ is a free parameter and $n_{0}=n(x=0)$. Finally, then, we have the particle density

$$
n(x)=n_{0}\left(1-2 \alpha R \tanh \left(\frac{x}{\lambda}\right)-\alpha \tanh ^{2}\left(\frac{x}{\lambda}\right)\right)
$$

which can be used to load the particles in the simulation code to create the initial state. Notice that

$$
\begin{aligned}
& n(x)_{x / \lambda \ll 0} n_{0}[1-\alpha(1-2 R)] \\
& n(x)_{x / \tilde{\lambda} \gg 0}^{\sim} n_{0}[1-\alpha(1+2 R)]
\end{aligned}
$$

and therefore

$$
\alpha \leq \frac{1}{1+2 R}
$$

to keep the density nonnegative on the low density side. This last condition limits the energy density of the field relative to that of the particles. Since we must have $R>1$ to avoid a null in the magnetic field, $\alpha \leq 1 / 3$.

[13] All variables in the DF simulation code are dimensionless. To relate their values to a dimensional environment, two fundamental parameters must be specified, $n_{0}$ and $B_{0}$. Both of these relate to the initial state of the simulation. Assuming no dependence on the $z$ coordinate, the particle density (equal for ions and electrons) at the central position in $x$ within the current sheet is given by $n_{0}$ and the magnetic field strength at the same position and time is $R B_{0}$. In applications to observed DF data, the field strength and particle density should be set to values observed in the central plasma sheet portion of the DF current sheet. Using the symbol * to denote dimensionless simulation variables, then the dimensional density $n=n_{0} n^{*}$ and, similarly, the magnetic field $\mathbf{B}=B_{0} \mathbf{B}^{*}$, time $t^{*}=\omega_{i c} t$ in which $\omega_{i c}$ is the ion cyclotron frequency in the $B_{0}$ field 


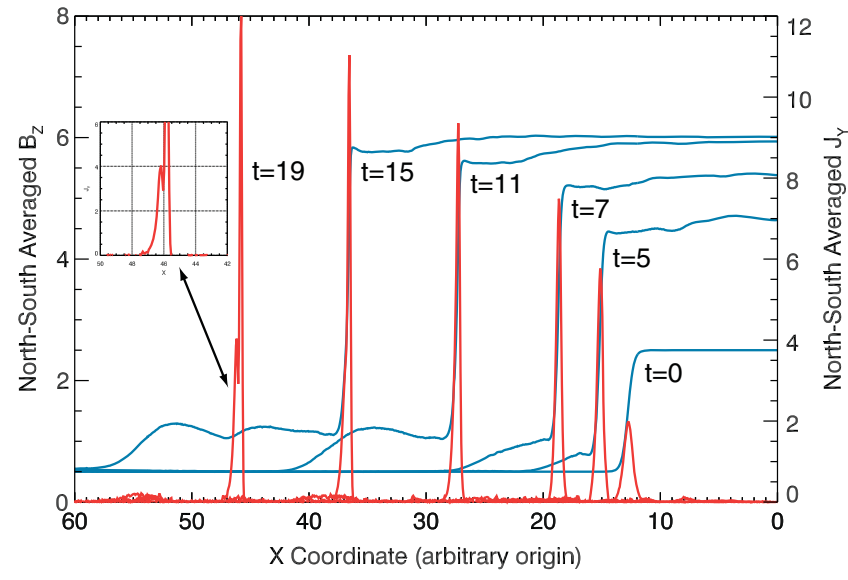

Figure 2. $B_{z}$ at indicated times (blue). (Curve at $t=19$ has been eliminated for clarity. $B_{z}$ strength in back of current sheet does not vary significantly from $t=15$ to $t=19$.) Dawn-dusk current density at indicated times (red). All curves show north-south averages over $z$.

strength, lengths are normalized with respect to the ion inertial length $d_{i}=c / \omega_{p i}=c\left(4 \pi e^{2} n_{0} / m_{i}\right)^{-1 / 2}$ (cgs units), particle and moment velocities are expressed as $\mathbf{v}=V_{A 0} \mathbf{v}^{*}$, in which $V_{A 0}$ is the Alfvén speed computed using the ion mass and $n_{0}$ and $B_{0}$ for the density and field strength, and the electric field $\mathbf{E}=V_{A 0} B_{0} \mathbf{E}^{*}$. The dimensional and dimensionless ion and electron pressure tensors are related through $\mathbf{P}=\left(B_{0}^{2} / 4 \pi\right) \mathbf{P}^{*}$ and the temperatures through $T^{*}=\left(4 \pi / B_{0}^{2}\right) n_{0} k T$.

[14] We have used the $2 \frac{1}{2}$-dimensional electromagnetic PIC code described in Hesse et al. [1999], modified to incorporate the open boundary conditions [Klimas et al., 2008, 2010, 2012]. The electromagnetic fields are integrated implicitly to avoid the Courant constraint on the propagation of light waves. The simulation discussed here (denoted DF-1 in the following) was initialized with approximately $4 \times 10^{8}$ particles on a $1025 \times 625$ grid in the $x \times z$ directions. The system size is $L_{x}=65.6 d_{i}$ by $L_{z}=40 d_{i}$. Two particle species, one of ions and one of electrons, with mass ratio $m_{i} / m_{e}=25$ were included. As a matter of simplicity in this first-of-its-kind simulation, particle temperatures were initialized with $T_{i}=T_{e}$. This choice is in keeping with the intent to drive the DF long enough to induce a loss of memory of the initial state of the simulated plasma. We show below, however, that the simulated DF propagates at super Alfvénic speed and, consequently, interacts with upstream plasma that remains unchanged in the initial state. In future studies, we will initialize with the more typical magnetotail $T_{i} \geq 5 T_{e}$. It will be important to explore the significance of this change. Using the electron cyclotron frequency $\omega_{e c}=e B_{0} / m_{e} c$ and the electron plasma frequency $\omega_{e p}=\left(4 \pi e^{2} n_{0} / m_{e}\right)^{1 / 2}$, we set $\omega_{e p} / \omega_{e c}=4$. To ensure particle energy conservation, very small integration time steps were used; $\Delta t=0.0005$ in the dimensionless time defined in the preceding paragraph. The initial current sheet thickness was set to $0.5 d_{i}$, the parameter $R=1.5$, and $\alpha=0.2$. The simulation was driven through the application of an outof-plane electric field component $E_{y}$ at the inflow boundary. This was ramped up from its initial value, 0 , to its final value, 15 , over the time interval, $t=0-5$.

[15] Charge conservation is guaranteed by an iterative application of a Langdon-Marder type correction [Langdon, 1992] to the electric field at each time step. At the open boundaries, we apply a gradient free boundary condition on the out-of-plane electric field component, set the normal component to zero, and we leave the $z$-component unchanged from the previous time step. Then, the Langdon-Marder correction is applied to correct the electric field over the entire grid, including at the boundaries. For the magnetic field at the open boundaries, we set the out-of-plane magnetic field component to zero. At each step in the iterative implicit field solver, we set the $z$-component of the magnetic field to satisfy $\nabla \times \mathbf{B}=\mathbf{j}$ and then the normal component to satisfy $\nabla \cdot \mathbf{B}=\mathbf{0}$. This totality of particle and field boundary conditions generally leads to stable, quiet solutions with no trace of charge buildup near the boundaries or anywhere else on the computational grid.

\section{Simulation Overview}

\subsection{Dawn-Dusk Current Density and $B_{z}$ Strength}

[16] Figure 2 provides an overview of the magnetic field and cross-tail current density encompassing most of the DF-1 simulation, which evolved from $t=0$ to $t=20$. For this figure and for the remainder of this paper, quantities denoted as north-south averaged will have been averaged over the full range of the $z$-coordinate. It can be seen that both the strength of the field jump at the current sheet and the strength and width of the current sheet evolve considerably under the influence of the driver field. Notice the development of a precursor, a pileup region, in the field strength. The simulation was stopped as the current sheet approached the outflow boundary and the majority of the pileup region had already exited. The continuing growth of the current sheet intensity, as well as the appearance of a bifurcation of the current sheet (inset), shows that the DF never came into equilibrium with the driver. At $t=0$, the current sheet was stationary and then only accelerated in response to the driver field later. As it accelerated, a pileup region began to form and then grow. Late in the simulation, the pileup region extended too far in front of the current sheet to be a valid model of the phenomenon in the magnetotail. This growth is a direct consequence of the combination of periodic boundary conditions in the north-south directions plus the lack of an $x$ component in

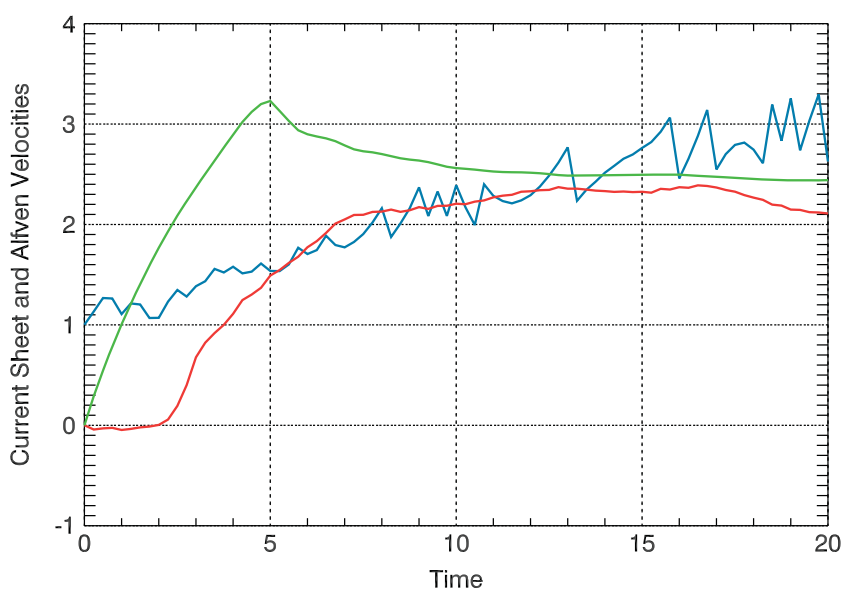

Figure 3. Plasma inflow speed at inflow boundary (green). Propagation speed of current sheet peak (red). Local Alfvén speed at the position of the current sheet peak (blue). 


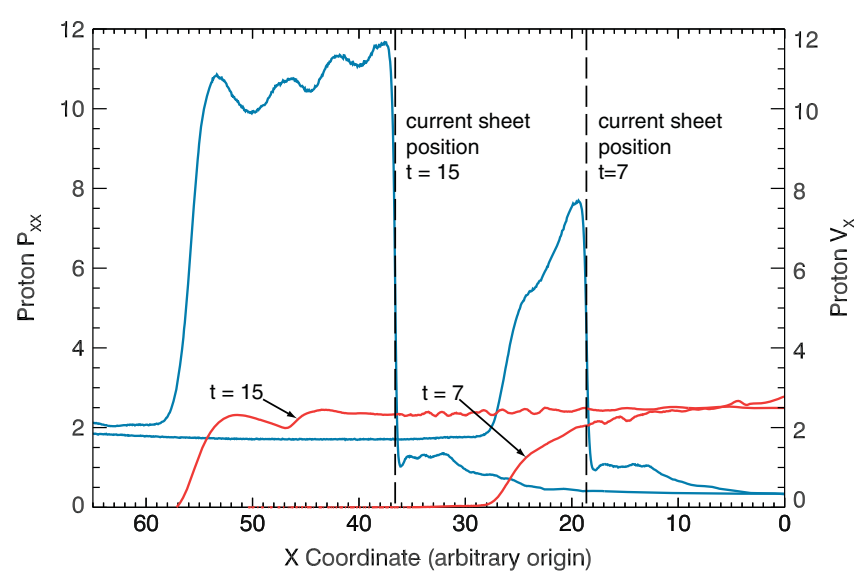

Figure 4. Showing the development of the pileup region at $t=7$ and $t=15$. Vertical dashed lines show the positions of the current sheet at these times. Ion pressure tensor component $P_{x x}$ (blue). Ion earthward flow speed (red). All quantities north-south averaged.

the initial magnetic field configuration. Plasma remained trapped in front of the propagating current sheet with no escape route, as in the magnetotail. In the next section, we show that by $t=7$ most of the acceleration of the current sheet had finished. From then until approximately $t=13$, the simulated pileup region remained an acceptable model of the magnetospheric counterpart. We have used simulation output from before and after those times in limited ways discussed below but our conclusions concerning the properties of the simulated pileup region are limited to its behavior in the interval $7 \leq t \leq 13$.

\subsection{Flow and Alfvén Speeds}

[17] Plasma was driven into the simulation domain through the imposition of a uniform out-of-plane electric field at the boundary. The inflow speed is given by $E_{y} / B_{z}$ at the boundary. The green curve in Figure 3 shows this inflow speed. The initial growth in speed up to $t=5$ is a consequence of the ramping up of $E_{y}$ during that interval. The following slow decline, with asymptote somewhat above 2 , reflects the growth with increasing time in $B_{z}$ at the boundary, which is evident in Figure 2. The red curve shows the speed at which the peak of the current sheet traveled toward the outflow boundary. After some delay while the driver field propagated to its position, the current sheet accelerated rapidly. The decline in this speed near the end of the simulation would be temporary in a longer simulation; the red and green curves must eventually come into agreement. The blue curve shows the evolution of the local Alfvén speed at the position of the current sheet peak. The large jumps in this local speed are due to the combination of a very steep gradient in $B_{z}$ strength at the current sheet position and a discrete grid on which to measure the strength. The local Alfvén speed appears to grow approximately linearly with time, although the noise near the end of the simulation admits the possibility that the speed may be saturating, as might be expected from the apparent saturation of the $B_{z}$ strength in back of the current sheet evident in Figure 2. With this proviso, we find that both current sheet speed and the local Alfvén speed self-adjust to approximate equality, in agreement with the observations of Hwang et al. [2011] in the central plasma sheet.

\subsection{Pressure and Flow Speed in Pileup Region}

[18] The compression, or pileup, region preceding the arrival of a DF current sheet is typically detected in the growth of the plasma pressure and flow speed before the arrival of the current sheet [Runov et al., 2011b, 2011a; Sergeev et al., 2012]. Figure 4 shows snapshots at two times of the ion pressure tensor component $P_{x x}$ and the ion flow speed $V_{x}$ taken from the DF-1 simulation. The positions of the current sheet at these two times are indicated by the vertical dashed lines. It can be seen that the spatial extents of both the pressure and flow speed precursors increase with increasing time while the pressure (and density, not shown) increases in amplitude as well. The relatively low pressure in back of the DF is due to the low particle density in that region. At the later time, the DF and local plasma in front and in back of the DF all move together at the same speed, in agreement with recent THEMIS spacecraft observations [Runov et al., 2009; Sergeev et al., 2009; Zhou et al., 2011]. At the earlier time, this is not exactly true but it can be seen from Figure 3 that the DF was still being accelerated somewhat at that time.

\subsection{Current Sheet Details}

[19] Observed DF current sheet thicknesses are typically of the order of 1-3 thermal ion gyroradius or ion inertial lengths (see Sergeev et al. [2012] and references therein). Panel (a) of Figure 5 provides a comparison of these length scales, at

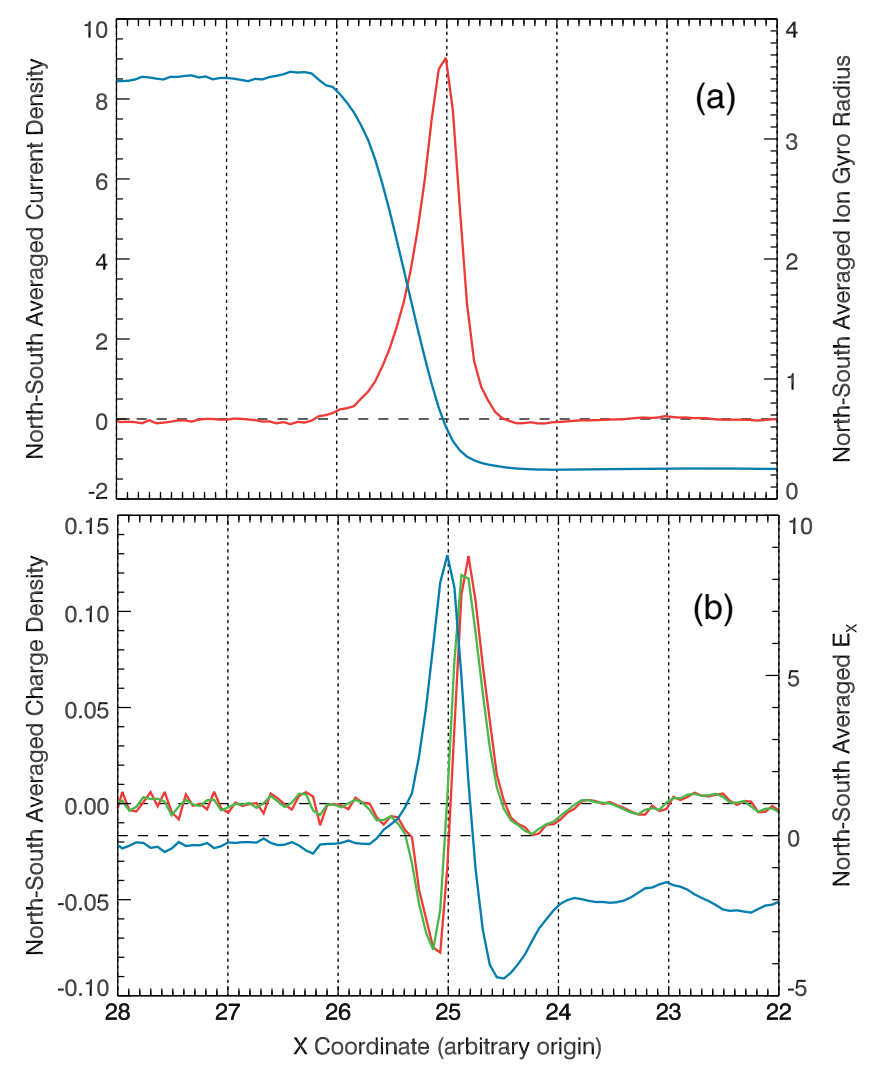

Figure 5. (a) Current sheet density (red). Thermal proton gyroradius in current sheet neighborhood (blue). (b) Charge density (red). Normal electric field component $E_{x}$ (blue). $\partial E_{x} / \partial x$ (green). 


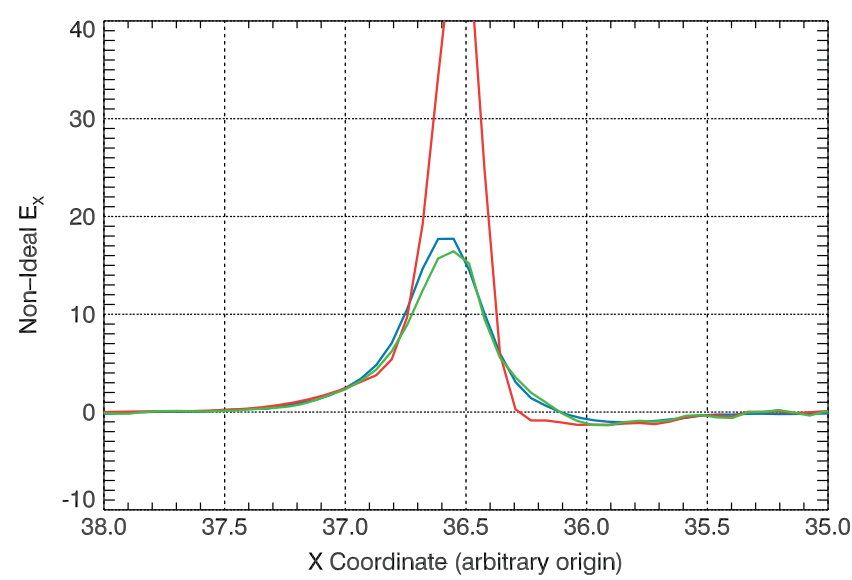

Figure 6. Nonideal $E_{x}^{*}=E_{\mathbf{x}}+\left[\mathbf{V}_{e} \times \mathbf{B}\right]_{x}$ (blue). Hall contribution to $E_{x}^{*}$ from (8) (red). Summed Hall and pressure gradient contributions from (8) (green).

$t=10$, for the DF-1 simulation. The $x$ coordinate is measured in units of the proton inertial length, computed using the proton density in the current sheet at $t=0$. Since the proton density in the current sheet does not change significantly over the entire simulation, the $x$ coordinate is effectively measured in units of the local ion inertial length at this time. The red curve in panel (a) gives a detailed view of the current sheet density. Depending on how it is measured, it can be seen that the current sheet thickness is of the order of a proton inertial length, or less. The blue curve shows the thermal proton gyroradius in the neighborhood of the current sheet. Because of the jump in field strength at the position of the current sheet, the gyro radius varies from $\simeq 3.5$ in front of the current sheet to $\simeq 0.3$ in back. At the position of the current sheet peak, the gyro radius is $\simeq 0.7$. Thus, we find that the inertial length, gyroradius, and current sheet thickness are all approximately equal.

[20] The steep growth in magnetic field strength at the current sheet position acts as a barrier that prevents the denser upstream plasma from entering the downstream region. Because the upstream proton gyro radii are comparable to the thickness of the current sheet, they are able to penetrate the magnetic barrier at the current sheet to a depth that is comparable to the current sheet thickness while the electrons are turned back more quickly. The consequent separation of electrons and protons leads to a layer of charge coincident with the position of the current sheet. The red curve in panel (b) of Figure 5 shows this charge distribution at $t=10$ in the DF-1 simulation. The negative peak to the left gives an indication of the penetration depth of the electrons while the positive peak to the right indicates the proton penetration depth. Consistent with the charge distribution, an earthward directed electric field $E_{x}$ is produced in a thin layer at the current sheet position. The blue curve in panel (b) illustrates this field. The positive pulse joins the positive and negative charge layers and is effectively shielded from the upstream region. The positive charge layer is unshielded to the right, leading to a tailward directed $E_{x}$ in the downstream region. The green curve in panel (b) is the result $\partial E_{x} / \partial x$, confirming that the field is the direct result of the separation of electrons and protons at the magnetic barrier. Consequently, $E_{x}$ might be considered a Hall type field.
[21] DF observations often exhibit a strong earthward directed Hall type electric field normal to and localized at the current sheet [Zhou et al., 2009; Runov et al., 2011a; Sergeev et al., 2012]. Zhou et al. [2009] have discussed a THEMIS event that exhibited a strongly peaked earthward directed DC electric field normal to the DF in the plasma rest frame, i.e., a DC peak in the nonideal $E_{x}^{*}=E_{x}+(\mathbf{V} \times \mathbf{B})_{x}$ component. They found that the peak could be very well fit by the Hall term $(\mathbf{j} \times \mathbf{B})_{x} / n e$ and, thus, concluded that the DC field was a Hall field. Runov et al. [2011a], however, in a superposed epoch study have found that the median $E_{x}^{*}$ is well explained by the Hall term in front of the DF but not in back. For the DF simulation under discussion here, we find that the electric field of Figure $5 \mathrm{~b}$ has two equally important contributions to its nonideal part, the Hall contribution and an opposing electron pressure gradient contribution.

[22] The nonideal corrections to the $\mathbf{V} \times \mathbf{B}$ drift contribution to $E_{x}$ can be obtained from the $x$-component of the dimensionless electron or ion momentum equations. Neglecting other than the $B_{z}$ component of the magnetic field, these can be written

$$
\begin{aligned}
E_{x}^{*}= & E_{x}+\left[\mathbf{V}_{\alpha} \times \mathbf{B}\right]_{x} \simeq E_{x}+V_{\alpha y} B_{z} \\
& \simeq \frac{1}{n_{\alpha} q_{\alpha}}\left[\frac{\partial}{\partial t}\left(m_{\alpha} n_{\alpha} V_{\alpha x}\right)+\frac{\partial}{\partial x}\left(m_{\alpha} n_{\alpha} V_{\alpha x} V_{\alpha x}+P_{x x \alpha}\right)\right]
\end{aligned}
$$

in which $\mathbf{E}^{*}$ is the nonideal portion of the electric field, the symbol $\alpha$ stands for either electron or ion quantities with $q_{\alpha}= \pm 1$, and $\mathbf{P}_{\alpha}$ is the electron or ion pressure tensor. For electrons in the DF simulation, we find that the thermal pressure gradient term on the right side of (7) dominates strongly. Thus, assuming $n_{i}=n_{e}$, we can write

$$
E_{x}^{*} \simeq \frac{j_{y} B_{z}}{n_{i}}-\frac{1}{n_{e}} \frac{\partial}{\partial x} P_{x x e}
$$

[23] The blue curve of Figure 6 shows the nonideal component of the $E_{x}$ illustrated in Figure 5. The contribution of the Hall term on the right side of (8) to $E_{x}^{*}$ is shown by the red curve; it peaks at approximately the value 50 . The summed contributions of both terms on the right side of (8) are given

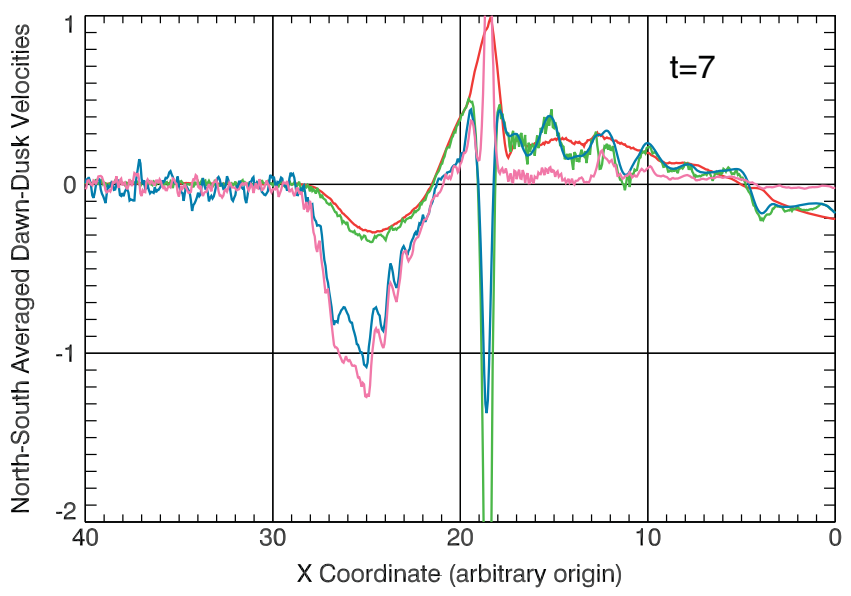

Figure 7. Ion $V_{y}$ (red). Electron $V_{y}$ (green). $\mathbf{E} \times \mathbf{B}$ drift velocity (blue). Electron $V_{y}$ with pressure gradient correction (magenta). 

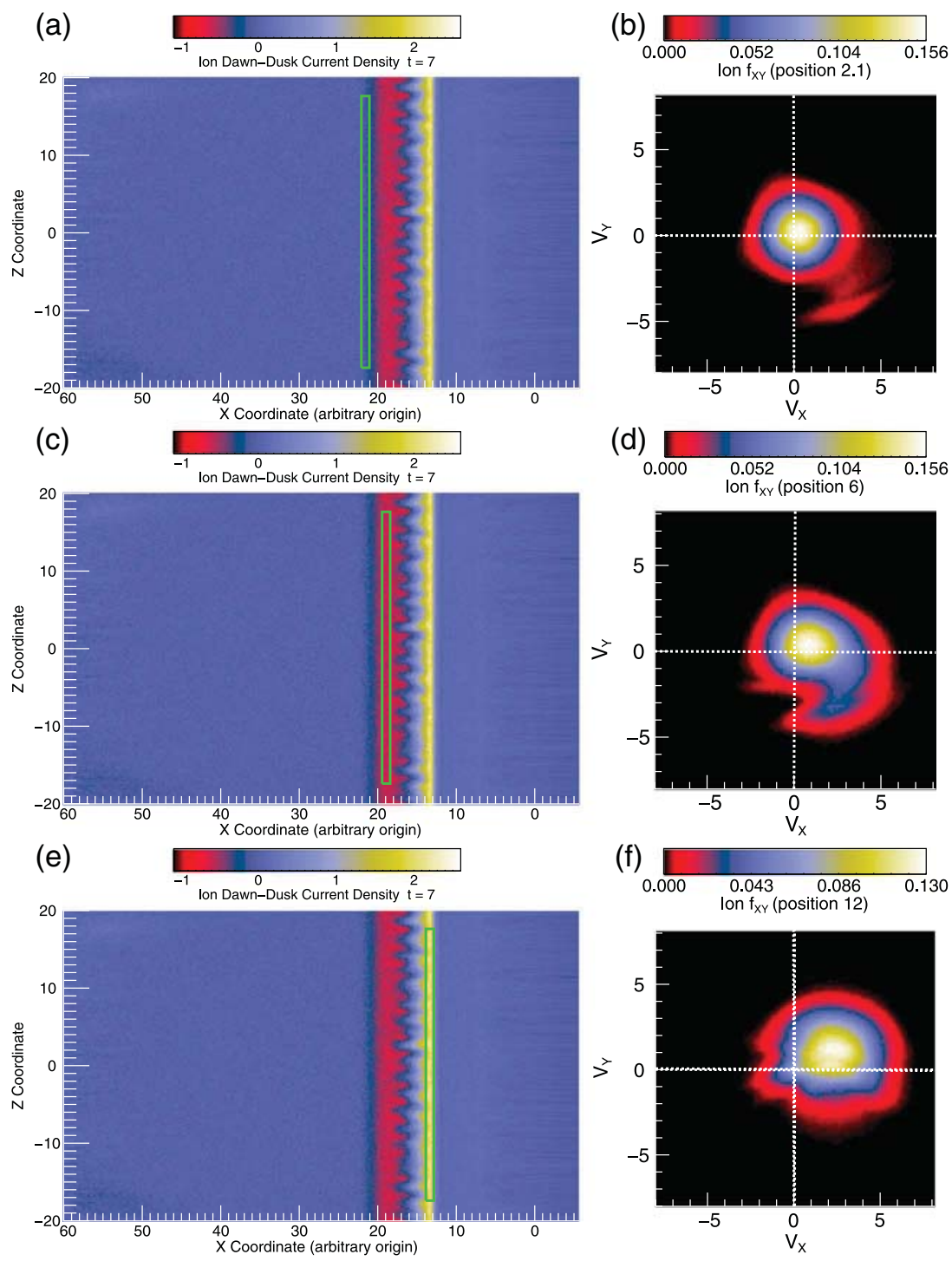

Figure 8. Left column: Ion dawn-dusk current distributions at $t=7$. Right column: Reduced ion perpendicular velocity distributions for particles collected from regions indicated by green rectangles superposed on current distributions to their left.

by the green curve. Since it is precisely the difference between $n_{i}$ and $n_{e}$ that is responsible for generating $E_{x}$, we have confirmed that this result is insensitive to the choice of $n_{i}$ or $n_{e}$ in the denominator of the Hall term, or even to the choice $\left(j_{y i} / n_{i}\right)+\left(j_{y e} / n_{e}\right)=V_{i y}-V_{e y}$. Thus, we conclude that in this DF simulation the nonideal electric field normal to the current sheet contains a large Hall contribution but also an almost equally large, and opposing, electron pressure gradient contribution.

\subsection{Dawnward ion and Electron Fluxes in Pileup Region}

[24] In a pileup region, near the magnetotail neutral sheet, ion fluxes are generally observed in the dawnward direction [Hwang et al., 2011; Zhou et al., 2011, 2012b]. Zhou et al. [2012b] have explained this behavior using the theory of Büchner and Zelenyi [1989] for particle trajectories in the magnetotail. From this theory, they were able to show that, after being reflected by the DF, only ions that quickly turn dawnward with reduced earthward speed can remain near
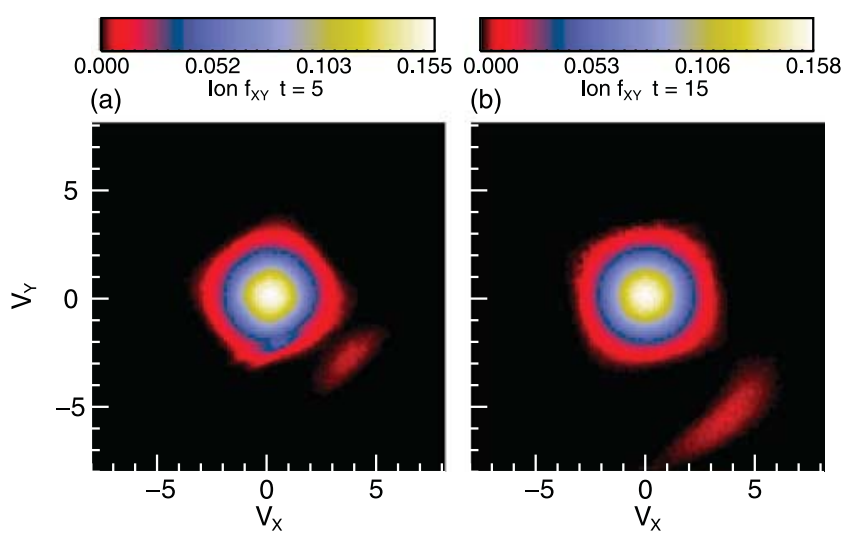

(b)

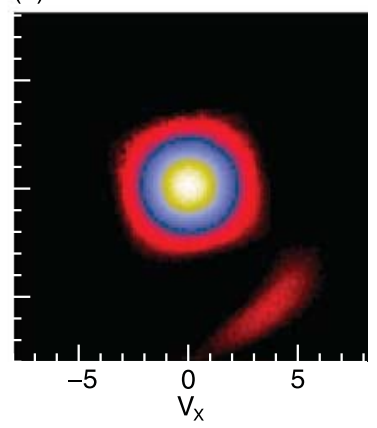

Figure 9. Two reduced ion velocity distributions just in front of the pileup region at (a) $t=5$ and (b) $t=15$. 


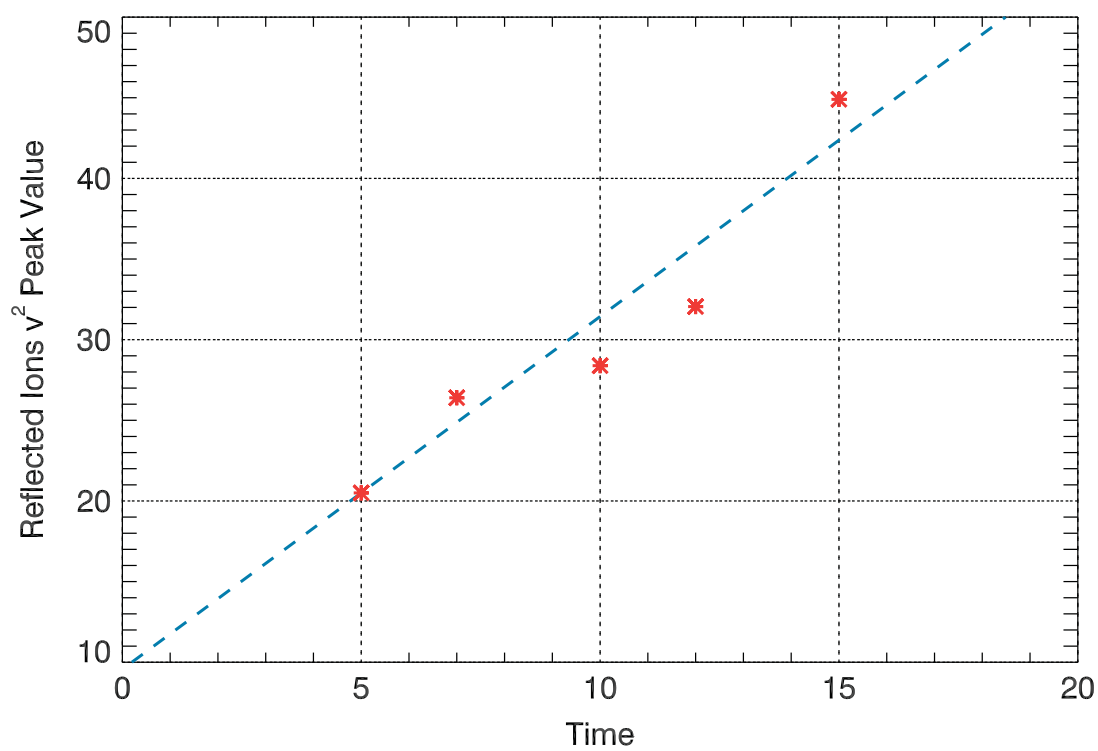

Figure 10. At selected times, $v^{2}$ values at peaks in reflected ion velocity distributions (red). Least squares linear fit (blue).

the neutral sheet; other ions that retain high earthward speed must move toward the PSBL. The result is a preponderance of dawnward moving ions near the neutral sheet in the pileup region.

[25] In the results of the DF-1 simulation, we find a similar dawnward ion drift in the pileup region. Below, we show that this drift is the expression in the velocity moment of the motion of a group of gyrophase bunched reflected ions as they begin to gyrate about the $B_{z}$ field after interacting with the DF. This behavior appears in the ion velocity moment component $V_{y}$ in the neighborhood of the DF, as shown by the red curve of Figure 7 at $t=7$. The negative excursion, indicating dawnward motion in the pileup region on the earthward side of the current sheet is evident. Since, as we show below, the electrons do not participate in the gyrophase bunched motion of the ions, it is somewhat surprising that the electron cross-tail drift in the pileup region, as shown by the green curve of Figure 7, is almost identical to that of the ions. The small difference that can be seen in the figure will be shown due to wave activity in the pileup region that involves local current. The blue curve of Figure 7 shows the cross-tail $\mathbf{E} \times \mathbf{B}$ drift speed. It can be seen that, despite the fact that the ions and electrons are drifting at essentially the same speeds, they do not drift in agreement with the $\mathbf{E} \times \mathbf{B}$ drift. For the electrons, this disagreement can be understood as due to the important thermal pressure gradient term discussed in the preceding paragraph. Retaining this term on the right side of (7), we have

$$
V_{e y}+\frac{1}{n_{e} B_{z}} \frac{\partial}{\partial x} P_{x x e}=-\frac{E_{x}}{B_{z}}
$$

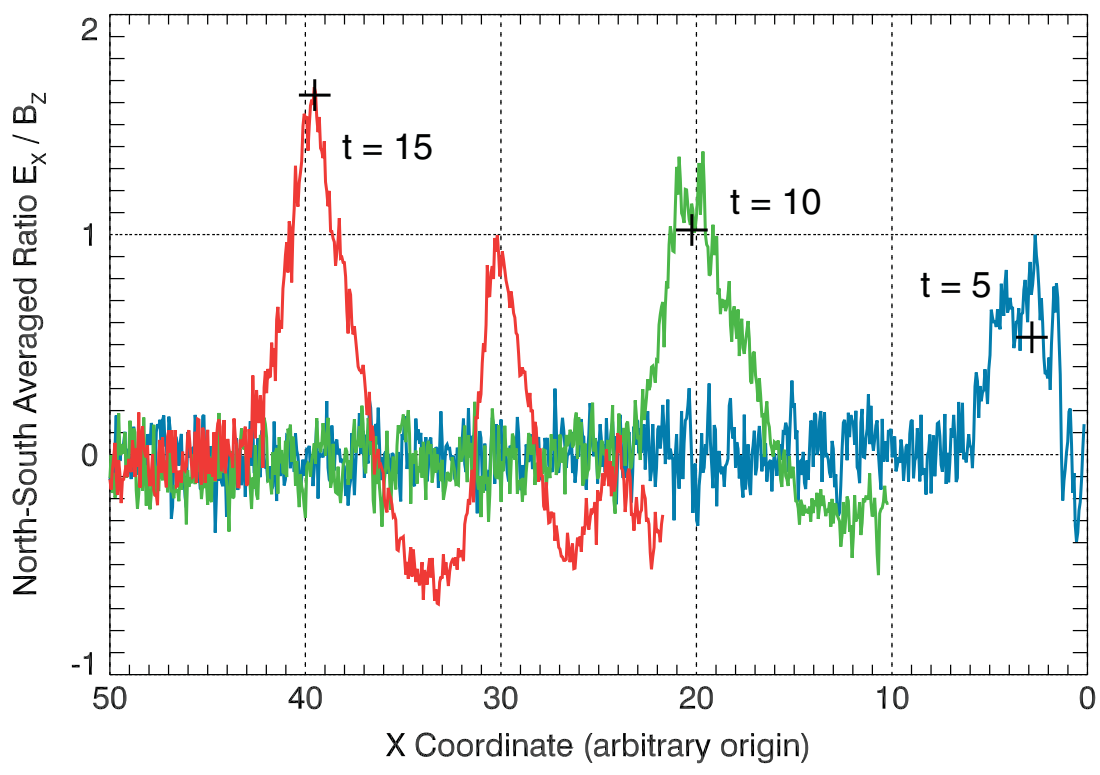

Figure 11. Selected portions of the north-south averaged ratios at $t=15$ (red), $t=10$ (green), and $t=5$ (blue). 


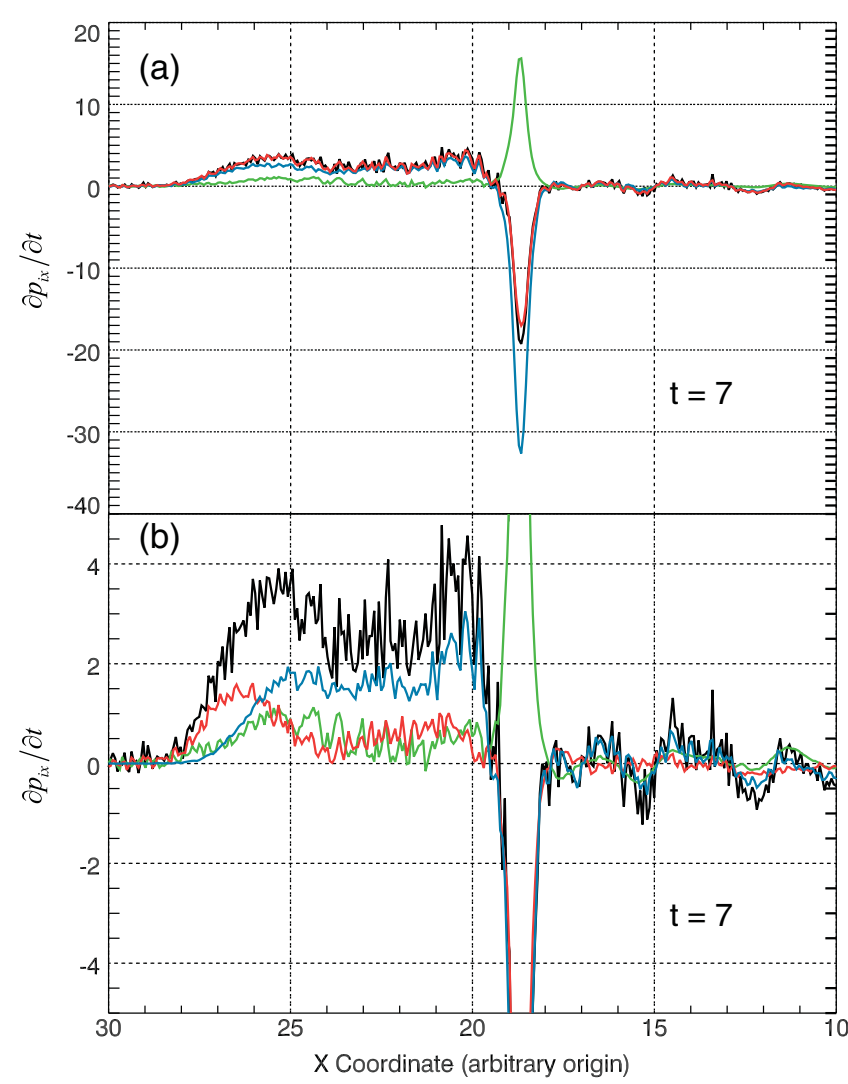

Figure 12. (a) Time derivative of ion momentum as a function of position $x$ (black). Total force density obtained through evaluation of all terms in the right-hand side of (13) (red). Electromagnetic force density (green). Pressure gradient force density (blue). (b) Time derivative of ion momentum as a function of position $x$ (black). Electromagnetic force density (green). Thermal pressure gradient force density (red). Dynamic pressure gradient force density (blue). All at $t=7$.

[26] The magenta curve of Figure 7 shows an evaluation of the left side of (9), in good agreement with the $\mathbf{E} \times \mathbf{B}$ drift, i.e., the right side of (9). We have found similar results for the ion drifts. However, in that case, all of the terms on the right side of (7) play important roles and none can be neglected.

[27] We are unaware of any published observations of dawnward electron flux in the pileup region. From Figure 4 of Hwang et al. [2011], it can be seen that the ion dawnward drifts are $\sim 200 \mathrm{~km} / \mathrm{s}$. If the electron drift equaled this value, then it would have to be detected in a velocity distribution whose thermal width is typically several hundred times as large, a very difficult task. We suspect that this difficulty is the reason for the lack of published dawnward electron drift observations.

\section{Reflected Ions}

[28] Zhou et al. [2010, 2011, 2012a, 2012b] have shown that many observed properties of the DF pileup region can be explained using their particle tracing model, which invokes ion reflection and acceleration at the DF current sheet. The DF-1 simulation exhibits ion reflection and acceleration as well; however, as we show in this section, the interaction in this case is between ions and the pileup region itself, a possibility that is absent from the particle tracing model.

[29] The second column of Figure 8 contains examples of reduced (integrated over $v_{\|}=v_{z}$ ) ion perpendicular velocity distributions obtained from the DF- 1 simulation at $t=7$. The images of the first column are all identical; they show the dawn-dusk ion current distribution at this time. The dawnward ion drift discussed above produces the region of negative ion current indicated by the red band to the left of the primary current sheet. Superposed on each image is a green rectangle showing the region in the ion current distribution from which the ions of the corresponding velocity distribution to the right were obtained. All of the distributions are effectively averaged over $z$. The first distribution (b) was constructed using ions from a region just in front of the pileup region. It can be seen that it consists of an essentially gyrotropic core that is slightly displaced from zero drift, plus a gyrophase bunched group of energetic, reflected ions in the $\left(v_{x},-v_{y}\right)$ quadrant. The second distribution (d) shows that this group of reflected ions is strengthened deeper in the pileup region, producing, despite the shift of the distribution core toward the $+v_{y}$ direction, the net dawnward current. Finally, the third distribution (f), from the DF current sheet, is roughly gyrotropic, heated relative to the distribution in front of the pileup region, and significantly shifted in the $\left(v_{x}, v_{y}\right)$ direction to accommodate the earthward propagation of the duskward directed current sheet.

[30] The energy of the reflected ions in front of the pileup region can be estimated, thereby showing that the particles are reflected by a normal electric field in the earthward direction and not through an interaction with the current sheet. Figure 9 shows two reduced ion velocity distributions from positions just in front of the pileup region at two times, the first quite early in the simulation and the second quite late. The upward shift in speed of the reflected ions with increasing time is evident. We have estimated the speed of the reflected ions by finding the peak in the distribution of reflected ions. Estimates of $v^{2}$ obtained in this manner are shown in Figure 10 for selected times. The dashed blue line is a least-squares fit given by $9.56+2.19 \times t$.

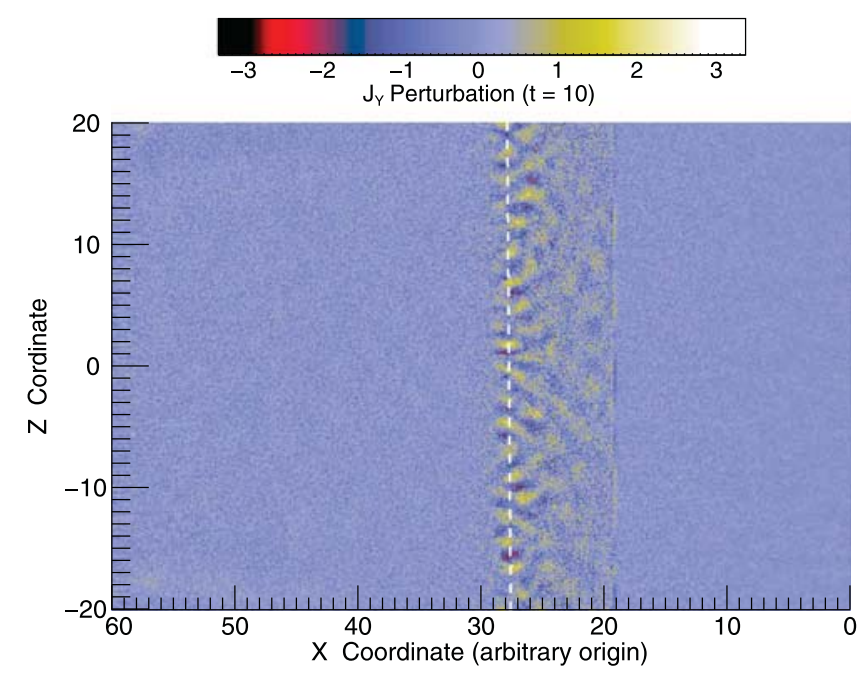

Figure 13. Dawn-dusk current distribution with northsouth averaged portion removed. Dashed white line marks position of maximum current filament wave activity. 


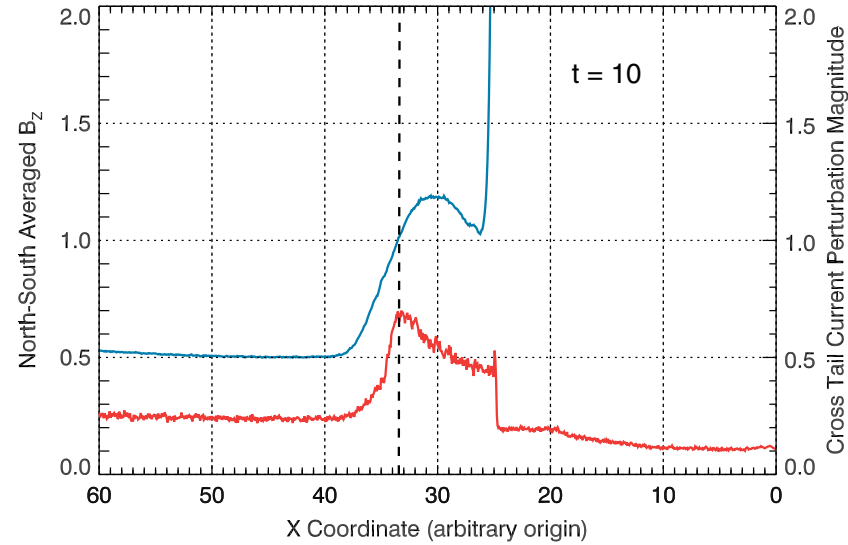

Figure 14. North-south averaged current filament wave magnitude (red). North-south averaged $B_{z}$ on expanded scale (blue).

[31] All of the points shown in Figure 10 were obtained from velocity distributions just upstream of the pileup region that contained, as in Figure 9, undisturbed core distributions at the same temperature. The increase in reflected ion $v^{2}$ shown in Figure 10 is a real increase; it does not reflect a change in the starting point of the reflected particles. Zhou et al. [2010] have shown that reflection of ions at the current sheet should increase the speed of the ions by approximately twice the speed of the moving current sheet. The approximately linear growth with time shown in Figure 10 is incompatible with the prediction of this mechanism given the evolution of the current sheet propagation speed shown in Figure 3. There, it can be seen that the propagation speed has essentially saturated by $t=7$. If this were the reflection mechanism at work, then the upward trend in Figure 10 should also cease at this time.

[32] Runov et al. [2011a] have noted, “... the dipolarization front may be described as a magnetopause of the energetic plasma intrusion into the quiet plasma sheet." In the following, we present evidence that suggests the leading edge of the pileup region behaves in some ways as a normal shock leading this magnetopause. In particular, the mechanism for reflecting and accelerating the gyrophase bunched ions at the leading edge may be similar to that which operates at Earth's bow shock to produce the upstream gyrophase bunched ions observed there [Eastman et al., 1981; Gurgiolo et al., 1981, 1983].

[33] In terms of the dimensionless variables in use here, and using north-south averaged electromagnetic field components, the motion of an ion upstream of the current sheet is governed by

$$
\begin{aligned}
& \frac{d v_{x}}{d \tau}=\frac{E_{x}}{B_{z}}+v_{y} \\
& \frac{d v_{y}}{d \tau}=-\left(v_{x}-V_{x}\right)
\end{aligned}
$$

with $\tau=B_{z} t$ and in which we have used $E_{y}=V_{x} B_{z}$ with $V_{x}$ being the $z$-averaged ion velocity moment component. With $v^{2}=v_{x}^{2}+v_{y}^{2}$ and treating the field components and $V_{x}$ as local constants, (10) yields

$$
\frac{1}{2} \frac{d v^{2}}{d \tau}=\frac{E_{x}}{B_{z}} v_{x}+V_{x} v_{y}
$$

[34] Leroy et al. [1981] have discussed the results of a 1-D hybrid simulation of Earth's bow shock in a perpendicular configuration. The foot region of their simulated shock is analogous to the leading edge of the pileup region in our DF-1 simulation. Their explanation for the reflected ions that they observed in the simulated shock foot region can be stated in terms of equation (10): They found that the electric field, $E_{x}$ (expressed as the gradient of a potential), was too weak to reflect the incoming ions. Ignoring the electric field in (10), we are left with ions gyrating in the $B_{z}$ field about the position $V_{x}$ in the velocity space. In their simulation, an ion that traveled from upstream into the foot region was turned around through this gyration motion while the electric field provided the extra acceleration necessary to convert it into a reflected ion traveling upstream. In the following, we explore the possibility that the reflected ions in the DF-1 simulation are produced in a similar manner.

[35] Consider a group of ions just upstream of the pileup region with the highest available $v_{x}\left(v_{y} \simeq 0\right)$ in the undisturbed thermal velocity distribution. From (10), it can be seen that as the pileup region engulfs them these ions will be accelerated in the negative $y$ direction and are therefore candidates for the gyrophase bunched dawnward drifting ions discussed in the preceding section. Figure 11 shows the ratio $E_{x} / B_{z}$ in the pileup region at three times in the DF-1 simulation. It can be seen that the ratio exhibits peaks at the leading edge of the pileup region. We will assume that, as in the Leroy et al. [1981] simulation, the group of high $v_{x}$ ions are reflected through their gyro motion in the pileup region $B_{z}$ and accelerated through their interactions with these peaks. We will assume that the interactions last for as long as it takes to turn the ions around, approximately a half gyro period. Then we will find that the energy gained through these interactions is consistent with the strengths of these peaks.

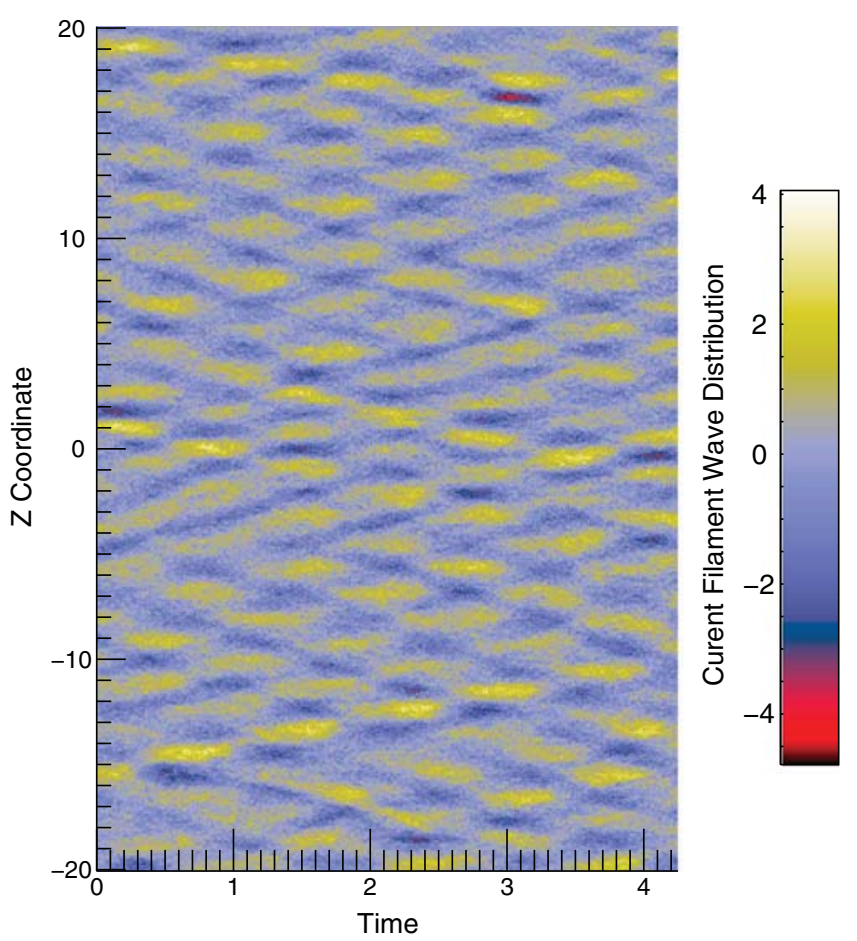

Figure 15. Evolution of dawn-dusk current density at position of maximum current filament wave activity. 

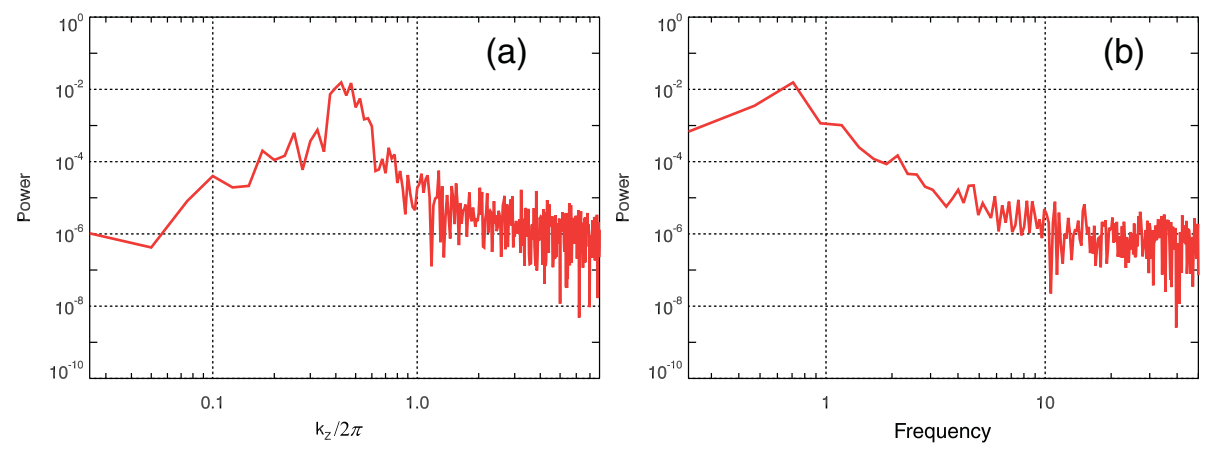

Figure 16. Current filament wave power distributions on cuts through the peak power position for $k_{z}>0$ in terms of (a) inverse wavelength and (b) frequency in units of $\omega_{c i}$.

[36] From (11), a rough estimate of the change in $v^{2}$ that occurs during one reflection interaction with $E_{x} / B_{z}$ can be obtained assuming that the interaction lasts a half gyro period, $\Delta \tau=\pi$. Then

$$
v^{2}=v^{2}(\text { init })+2 \pi\left(\frac{E_{x}}{B_{z}}\right) \overline{v_{x}}
$$

in which $\overline{v_{x}}$ is a measure, to be determined, of this particle velocity component during the interaction.

[37] Figure 11 shows the distribution of $E_{x} / B_{z}$ at $t=5$ (blue), $t=10$ (green), and $t=15$ (red). To avoid confusion, only the portions of the distributions on the leading side of the current sheet have been plotted. The green curve, for example, shows the extension (divided by $B_{z}$ ) into the pileup region of the negative $E_{x}$ on the upstream side of the $E_{x}$ peak shown in panel (b) of Figure 5. Each $E_{x} / B_{z}$ distribution is effectively zero in front of a leading peak that coincides (not shown) with the leading edge of the pileup region. The increase of the strength of this peak with increasing time is evident. If this peak is responsible for the reflection of ions at the leading edge of the pileup region, then its strength must be compatible with the measured growth of $v^{2}$ with time shown in Figure 10. We use the intercept at $t=0$ of the linear fit to the points on Figure 10 for $v^{2}($ init); i.e., we are assuming that in the limit $E_{x} / B_{z} \rightarrow 0, v^{2}$ remains at the value $v^{2}($ init $)$ and that value is given by the intercept value, $\simeq 10$. Further, we use $v$ (init) for $\overline{v_{x}}$. These choices are consistent with the particle lying initially on the outer fringes of the core velocity distributions shown, for example, in Figure 9. Since, from (11), maximum acceleration is given to the highest $v_{x}$ available, we should expect to find the origin of the gyrophase bunched accelerated ions at the highest speed fringe of the core velocity distribution. With these choices, we use (12) to solve for the strength of $E_{x} / B_{z}$ that a particle must interact with for a half gyro period to attain the $v^{2}$ value given by the linear fit of Figure 10 at the times, 5,10 , and 15 . Those strengths are indicated by the positions of the black crosses on Figure 11. It appears that this is a plausible explanation for the reflection and acceleration of ions at the leading edge of the pileup region; it does not involve reflection at the current sheet, as in the Zhou et al. [2010, 2011, 2012a, 2012b] model. Notice, from Figure 10, the energy of the reflected ions is increased by approximately a factor of 2.5 at $t=7$.

\section{Force Balance}

[38] Li et al. [2011], using an appropriate configuration of three of the THEMIS spacecraft, have studied changes in the total plasma pressure gradient and magnetic curvature force densities coincident with the arrival of a DF in the plasma sheet. Using the spacecraft configuration to model the
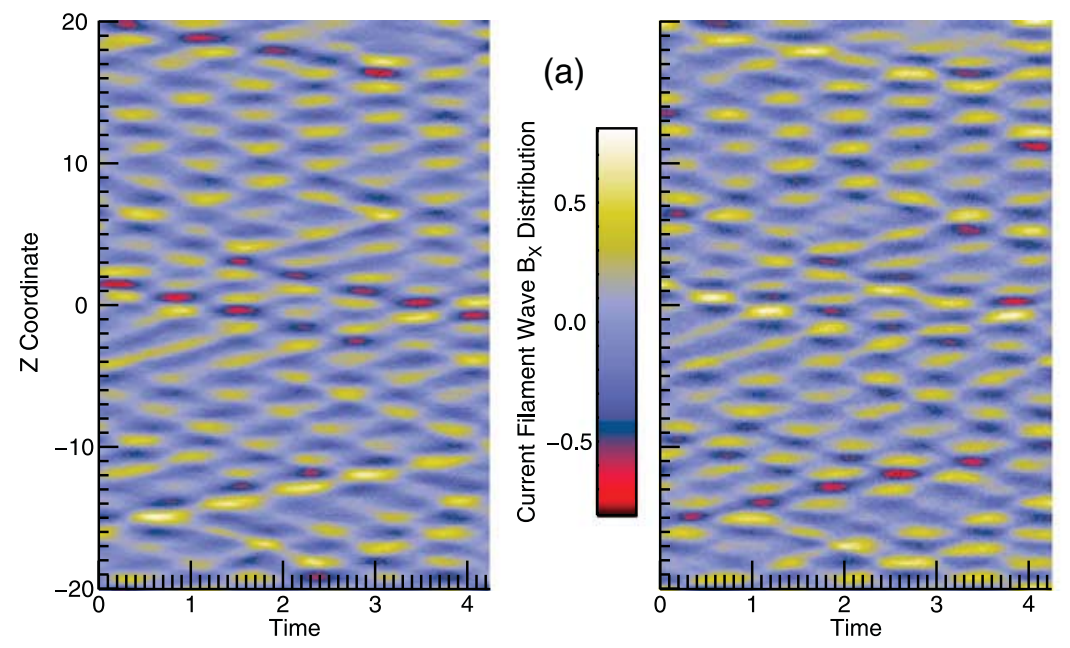

(b)

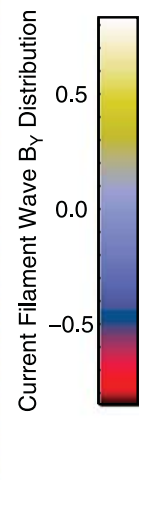

Figure 17. Evolution of (a) $B_{x}$ and (b) $B_{y}$ at position of maximum current filament wave activity. 


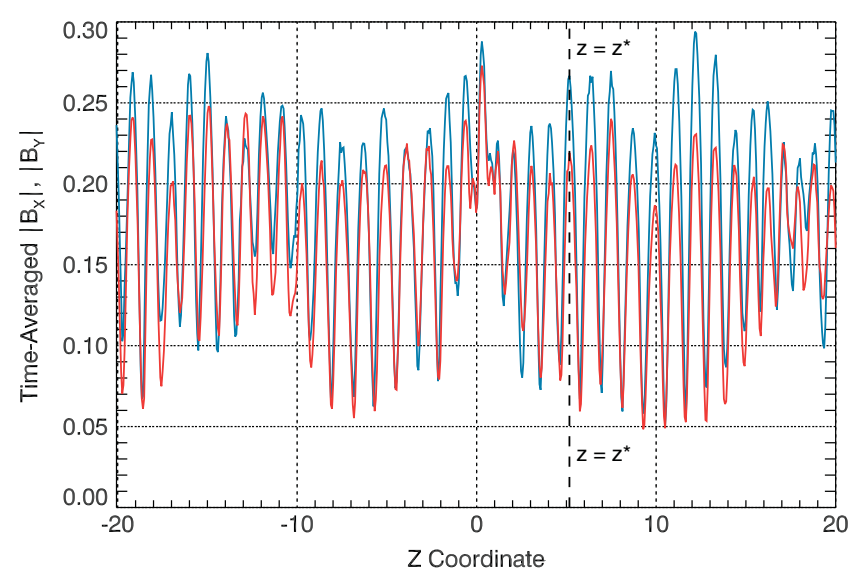

Figure 18. Time averages of the magnitudes of the magnetic field component perturbations shown in Figure 17. Time averaged $B_{x}$ wave amplitude (red). Time averaged $B_{y}$ wave amplitude (blue).

curvature of the magnetic field and assuming a constant propagation speed to determine spatial gradient scales, they were able to quite accurately predict changes in the plasma flow speed in the DF neighborhood. In back of the DF current sheet, they found that, while the curvature of the field lines decreased, the large increase in field strength led to an increase in curvature force that dominated the effects of the pressure gradient force. This aspect of their observations is modeled in the DF-1 simulation by the large jump in $B_{z}$ at the position of the current sheet. Li et al. found that the plasma in the pileup region was accelerated earthward by a reduction in the normally tailward directed pressure gradient force while the curvature force in this region remained relatively fixed. The undisturbed plasma in the DF-1 simulation does not contain a tailward pressure gradient force or a magnetic curvature force to balance it. Nevertheless, we do find that the disturbed pileup region plasma is accelerated earthward due primarily to an earthward directed pressure gradient force in that region.

[39] The $x$-component of the ion momentum $p_{i x}=$ $m_{i} n_{i} V_{i x}$ obeys

$$
\frac{\partial p_{i x}}{\partial t}=n_{i}\left[E_{x}+\left(\mathbf{V}_{i} \times \mathbf{B}\right)_{x}\right]-\frac{\partial}{\partial x}\left[m_{i} n_{i} V_{i x} V_{i x}+P_{x x i}\right]
$$

[40] There are two driving forces for changing the ion momentum. We will denote these as the electromagnetic force density (first term on the right side) and the pressure gradient force density (second term). The pressure gradient force contains two sources, the dynamic pressure (bulk velocity components) and the thermal ion pressure $\left(P_{x x i}\right)$. These can be evaluated directly from the DF-1 simulation.

[41] The black curve in panel (a) of Figure 12 shows a direct evaluation, at $t=7$, of the time derivative on the left side of (13). The red curve shows the agreement of a superposition of all of the contributions on the right side of (13). The peaks in this figure are at the position of the current sheet. In back of the current sheet, the approximately uniform plasma is moving earthward, leading to no significant change in the ion momentum. At the position of the current sheet, there is a net negative acceleration of the ions. The green curve shows the expected positive acceleration due to the electromagnetic (primarily magnetic) force, which is, however, overwhelmed by the negative peak in the pressure gradient force (blue curve). The negative peak can be understood through an examination of Figure 4 . There, it can be seen that the earthward flow speed is relatively constant across the current sheet position but the thermal pressure drops precipitously at that position due to the drop in ion density at the current sheet. The dynamic pressure behaves similarly. Thus, as the current sheet passes a fixed spatial position the ion momentum density drops suddenly, leading to the negative peak in Figure 12a.

[42] Panel (b) of Figure 12 shows some details of the contributions of the individual terms on the right side of (13) in the pileup region. Once again, the black curve shows the result of a direct evaluation of the time derivative on the left side of (13). It can be seen that the total force density leads to an approximately uniform growth rate for the ion momentum in the pileup region, i.e., an earthward force density. The green curve shows that the contribution of the electromagnetic force density is relatively minor, in agreement with the results of $\mathrm{Li}$ et al. [2011]. The red curve shows the contribution of the thermal pressure gradient force. The peak in this contribution at the leading edge of the pileup region reflects the energization of the reflected ions, leading to an increase in the temperature moment. The dynamic pressure gradient force dominates the remainder of the earthward acceleration in the pileup region.

[43] It is important to note that (13) and the discussion of the preceding paragraphs relate to force balance and momentum change at a fixed spatial position. Li et al. [2011], however, studied these issues in a reference frame moving with the local plasma through their choice of the convective derivative format of the MHD momentum equation. (13) can be transformed to that format to obtain

$$
m_{i} n_{i}\left(\frac{\partial V_{i x}}{\partial t}+V_{i x} \frac{\partial V_{i x}}{\partial x}\right)=n_{i}\left[E_{x}+\left(\mathbf{V}_{i} \times \mathbf{B}\right)_{x}\right]-\frac{\partial P_{x x i}}{\partial x}
$$

[44] From this perspective, the dynamic pressure gradient term vanishes leaving the electromagnetic and thermal pressure gradient force densities, both of which are unchanged by the transformation. Thus, ignoring the blue curve in panel (b) of Figure 12, it can be seen that the thermal pressure gradient dominates at the leading edge of the pileup region, the electromagnetic force density peaks up at a lower value just in back of the thermal peak, and deeper in the pileup region both the electromagnetic and thermal pressure gradient force densities are comparable and relatively weaker. Much of the earthward acceleration is provided by the thermal pressure gradient and electromagnetic force densities at the leading edge of the pileup region as plasma is gathered up there by the oncoming disturbance. In the preceding section, we have seen that the ion behavior in this region is highly dynamic and greatly influenced by the electric field as well as the magnetic field. It is not possible to reduce the electromagnetic term of (13) to MHD concepts generally but it is reasonable to think of a magnetic pressure gradient contribution at the leading edge of the pileup region where the field strength grows rapidly toward the interior of the region. To this degree, the DF-1 simulation results are consistent with those of $\mathrm{Li}$ et al. with an earthward total pressure gradient force density 


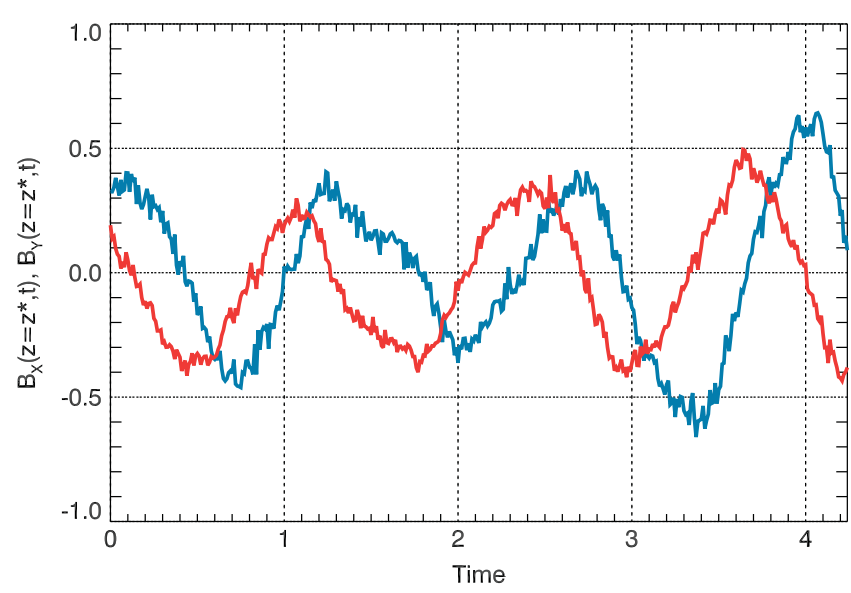

Figure 19. At $z=z^{*}$ in Figure 18, evolution of $B_{x}$ (red) and $B_{y}$ (blue) with time.

accelerating the pileup region in the simulation and with a reduction in the tailward total pressure gradient force density providing the acceleration in the magnetotail. More generally, without the assumption of a magnetic pressure gradient contribution to the electromagnetic term, it can be said that the DF-1 simulation results provide a somewhat generalized but compatible interpretation of the $\mathrm{Li}$ et al. results.

\section{Plasma Wave Activity in the Pileup Region}

[45] We are unaware of any reports of observed plasma wave activity in the upstream portion of a pileup region in the magnetotail. However, wave activity in this region is a persistent feature in our various DF simulations, including the DF-1 simulation. In this section, we describe the properties of these waves in some detail and discuss the possibility that the wave activity is present in the observational data but not noticed. It appears that the wave activity might be gradient driven by the rising pressure, field strength, and/or density at the leading edge of the pileup region and then strongly damped in back of the leading edge. There is also a possible relationship with the highly nongyrotropic ion velocity distributions in that region associated with the gyrophase bunched reflected ions. We have not identified the wave mode(s) involved.

\subsection{Current Filament Waves}

[46] One aspect of the wave activity at the leading edge of the pileup region in the DF-1 simulation is illustrated in Figure 13. The dawn-dusk current density has been averaged in $z$ and then the average removed to produce the perturbations in the current distribution exhibited in the figure. The DF current sheet at $x \simeq 19$ is essentially free of $z$-dependence and, so, it does not appear. The activity in the vicinity of the vertical dashed line, however, is unaffected since its $z$-average was originally negligible. A comparison with the color bar shows that the uniform blue color distributed over the majority of the figure corresponds to $J_{y}=0$. Regions with yellow color contain positive current density and regions with darker blue or even red contain negative current density. The disturbance consists of filaments of current whose directions oscillate with varying $z$; the wavelength in this direction is approximately 2 ion inertial lengths. The filaments grow in front of the dashed white line, which is moving earthward, peak at its position, and decay in back of it. Each time a filament grows, it is in the opposite direction from the one preceding it at its position in $z$. In that sense, the pattern oscillates in the $x$ direction also but the pattern is not visible at an instant in time due to its limited distribution in $x$.

[47] We have averaged the magnitude of the current filament waves over $z$. The result, at $t=10$, is shown by the red curve in Figure 14. The blue curve shows the rise in $B_{z}$ in the pileup region, leading to the sudden jump upward at the position of the current sheet. The extent of the pileup region at this time is shown by the extent of this region of increased $B_{z}$ in front of the current sheet. It can be seen that the strength of the wave activity exhibits a peak that is coincident with the $B_{z}$ gradient at the leading edge of the pileup region. This is a position of increasing density, pressure, and flow speed (see Figure 4) as well. It appears possible that the wave activity
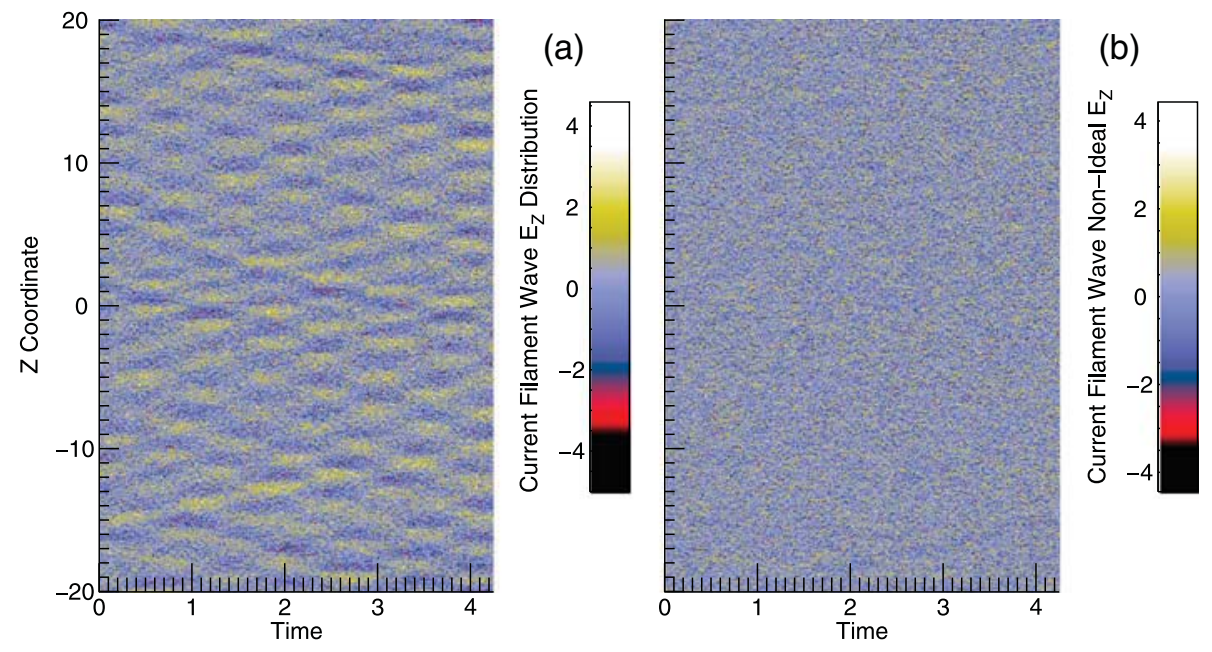

Figure 20. (a) Current filament wave activity in the $E_{z}$ electric field component at the peak wave amplitude position. (b) Nonideal z component, $E_{z}+V_{x} B_{y}$, at the same position. 

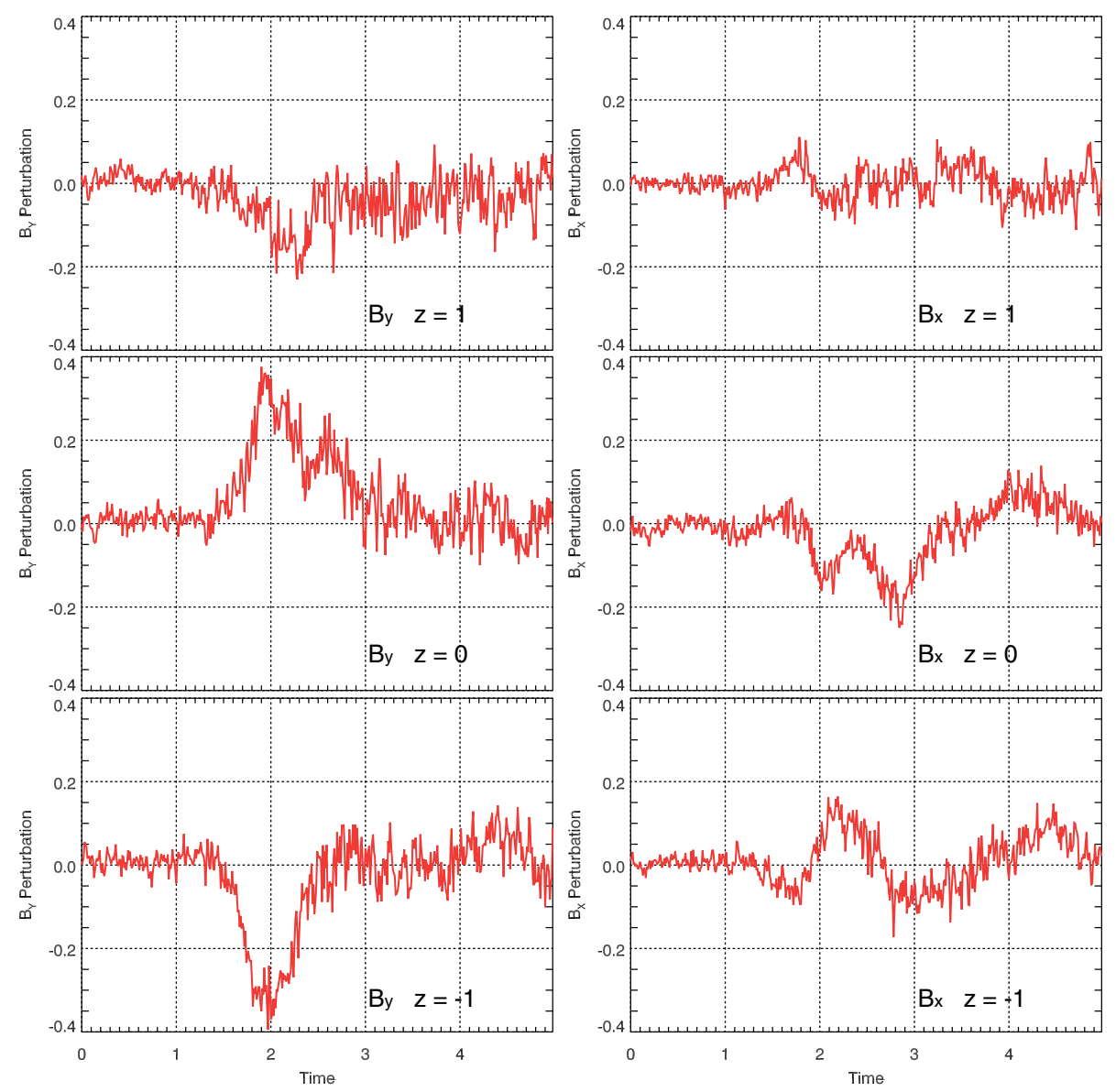

Figure 21. The passage of a current filament wave disturbance over a simulated spacecraft at three nearby positions. Left column: $B_{y}$ at (top) $z=1$, (middle) $z=0$, and (bottom) $z=-1$. Right column: $B_{x}$ at the same positions. Time measured in units of $\omega_{c i}^{-1}$.

is gradient driven. It should be noted, however, that the ion velocity distribution is significantly nongyrotropic at the leading edge of the pileup region due to the presence of the gyrophase bunched reflected ions, as shown in panels (a) and (b) of Figure 8. The reflected ions may be playing a role as well in exciting the wave activity.

[48] The black dashed line drawn on Figure 14 is at the position of the wave activity peak at this time. We have followed the position of this peak over the course of the simulation and have recorded the distribution in $z$ of various properties of the simulated plasma along this vertical line. Figure 15 shows the evolution with time of the dawn-dusk current distribution along this line over the time interval $t=10-14.26$. It can be seen that, at any fixed $z$, the current oscillates regularly for a total of approximately three wave periods over the time interval shown; the wave period is somewhat larger than $1 \omega_{c i}^{-1}$. At neighboring $z$-values, separated by approximately two ion inertial lengths, the waves are $180^{\circ}$ out of phase; the wavelength in the $z$ direction is roughly two ion inertial lengths. To evaluate these properties more precisely, we have carried out a $2 \mathrm{D}$ power spectral analysis of the distribution shown in Figure 15.

[49] We have found that the 2D current filament power distribution contains a single peak at period $1.4\left(\omega_{c i}^{-1}\right)$ and wavelength 2.4 ion inertial lengths for $k_{z}>0$ and period 1.4 $\left(\omega_{c i}^{-1}\right)$ and wavelength 2.0 ion inertial lengths for $k_{z}<0$. Figure 16 shows the power distributions on cuts through the peak power position for $k_{z}>0$. The distributions for $k_{z}<0$ are similar. The dimensional frequency corresponding

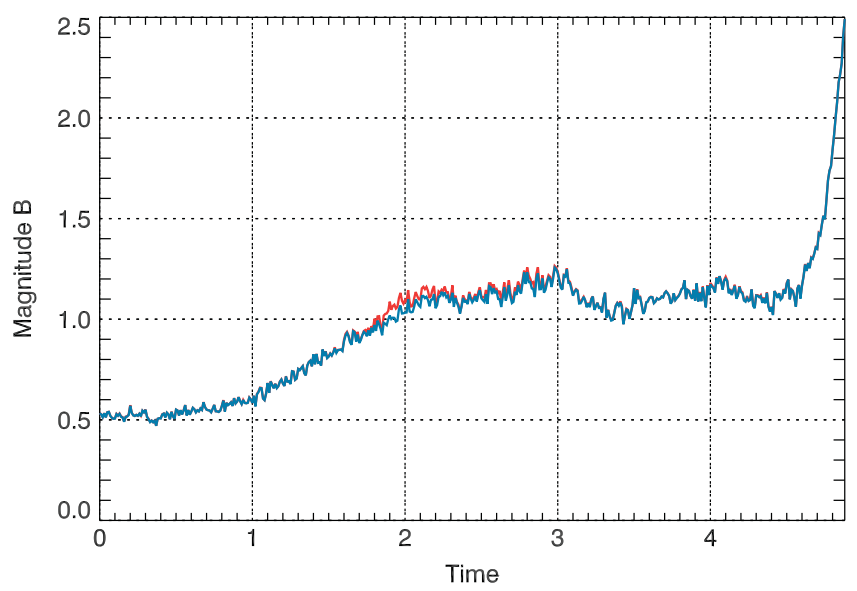

Figure 22. Magnetic field magnitude measured at the middle row spacecraft position of Figure $21(z=0)$. Field magnitude including wave perturbations (red). Field magnitude in the absence of the wave perturbations (blue). 
to period 1.4 is approximately $4.5 f_{c i}$, in which $f_{c i}$ is the ion cyclotron frequency.

[50] We have calculated the electron and ion betas, averaged over $z$, for various times throughout the DF-1 simulation. Throughout the pileup region $\beta_{i, e} \gg 1$ for all $t \geq 7$, when the current sheet acceleration has effectively ended. The current filament waves reside in high beta plasma.

\subsection{Magnetic Field Wave Activity}

[51] Figure 17 shows the evolution with time of the (panel a) $B_{x}$ and (panel b) $B_{y}$ field components along the line of maximum wave activity, constructed as discussed above. There is an analogous $B_{z}$ distribution but the disturbance amplitude is relatively small; it will be neglected in the following. The conclusions of this section are unaffected by this simplification.

[52] The field components evolve in a manner similar to that of the current filaments. At any $z$, each of the components oscillates in time at the same frequency as that of the current filaments. At neighboring $z$ values approximately 2 ion inertial lengths apart, the oscillations are $180^{\circ}$ out of phase. If this is the case, then there should be layers of no activity between the out-of-phase layers. Further, it appears as though one of the images is almost a copy of the other with a small shift in time between the two. These properties are examined further in the following.

[53] Figure 18 shows the result of taking the time average of the magnitudes of each of the field components shown in Figure 17 and plotting them together as functions of $z$ for comparison. Indeed, it can be seen that the activity in each component is layered with apparent nulls between the layers. We conclude from this observation that the waves in the magnetic field do not propagate along the background field, in the $z$ direction. If the waves did propagate in the $z$ direction, then the nulls would be smoothed over by the time averaging done to produce the figure.

[54] From Figure 18, it can be seen that the activity peaks of the two magnetic field components coincide quite precisely. In Figure 19, we examine the relationship between the two components at a position $z=z^{*}$ that coincides with one of the peaks; the results are typical of the behavior in any peak. It can be seen that the two components evolve with a well-defined relative phase relationship. We conclude that the waves are right handed elliptically (almost circularly) polarized with adjacent activity layers in $z$ maintaining a $180^{\circ}$ out-of-phase relationship.

\subsection{Electric Field Wave Activity}

[55] The pileup region plasma just in front of the current sheet moves at the speed of the current sheet. The position of the leading edge of the pileup region moves faster as new, initially stationary, plasma is added. Within the leading edge, the plasma flow speed exhibits a steep transition from stationary to the current sheet speed. It is in this transition region that the current filament waves peak.

[56] Panel (a) of Figure 20 shows that the $E_{z}$ component of the electric field participates in the current filament wave activity. The frequency, wavelength, and wave pattern are almost identical to those of the magnetic field components. Panel (b) shows the $z$ component of the nonideal electric field, $E_{z}+V_{x} B_{y}$ in which both $V_{x}$ and $B_{y}$ are measured at the wave activity peak position; the possible $V_{y} B_{x}$ contribution is negligible. The lack of a residual signature shows that the current filament waves are stationary in the local plasma; the waves move earthward with the local plasma propagation speed. We have found that the flow speed exhibits wave activity in this region also (not shown). These flow speed perturbations are included in the local plasma propagation speed.

\subsection{Detection}

[57] The current filament waves are a persistent feature of our driven DF simulations but there seems to be no observational counterpart in the magnetotail. To investigate this dichotomy, we simulate spacecraft observations of these waves by recording their signatures at various fixed positions as the wave front passes over. Figure 21 shows examples of the perpendicular $B_{x}$ and $B_{y}$ field components seen at three nearby positions spaced by one ion inertial length in the $z$ direction. This particular group of examples has been chosen because the wave signatures are atypically large.

[58] The combination of fast earthward propagation speed, narrow wavefield disturbance, and oscillation period commensurate with the disturbance passage time leads to a short-lived unpredictable disturbance at any of the observation points. Very small spatial displacements result in the appearance of unrelated signatures. No clear relationship between the evolution of the $B_{x}$ and $B_{y}$ field components is discernible.

[59] The amplitude of these disturbances is measured in units of the field strength in the current sheet at the beginning of the simulation, whose relationship to measured field values in the magnetotail is remote. Notice, however, from Figure 22 it can be seen that, in these same units, the strength of the field magnitude in the pileup region is close to one. Thus, in effect, the amplitudes of the disturbances are measured in units of the strength of the field magnitude in the pileup region.

[60] Using one unit of time as a rough estimate of the duration of the disturbances shown in Figure 21, if the field strength in the pileup region were $1 \mathrm{nT}$, then the dimensional duration would be $10 \mathrm{~s}$ and if the field strength were $10 \mathrm{nT}$, the duration would be $1 \mathrm{~s}$. For either case, however, the wave period would be commensurate with the passage of the disturbance.

[61] Figure 22 shows the magnetic field magnitude measured at the middle row spacecraft position of Figure 21 $(z=0)$. It can be seen that the entire pileup region, stretching from its initial growth at $t \simeq 0.4$ to the sudden rise signaling the approach of the current sheet, is observed. The red curve shows the magnitude including the contributions of the current filament wave activity and the blue curve shows the magnitude neglecting the wave activity. The differences in the two curves lie at the leading edge of the pileup region, as expected, while remaining almost indiscernible.

[62] Overall, we feel that the wave signatures shown here, if they were to exist in magnetotail field data, might not be easily recognized as indicating wave activity.

\section{Summary}

[63] We have discussed results from an ongoing driven, open-boundary PIC simulation study of the dipolarization front structure and propagation in Earth's plasma sheet. Initial results from the highly idealized DF-1 model are 
encouraging, indicating that further effort in this direction is warranted. Improvements, to better model the undisturbed plasma sheet in front of the DF, are apparent and in progress. These will be reported in a future publication.

[64] Several defining properties of observed DFs are reproduced by the DF-1 model. Under sufficiently strong driving, the current sheet achieves an earthward propagation speed of approximately the local Alfvén speed while retaining a coherent, numerically stable structure. The $B_{z}$ field strength in back of the current sheet and the current sheet intensity and thickness all self adjust under the influence of the driver at the inflow boundary such that the current sheet thickness, thermal ion gyroradius at the current sheet, and ion inertial length evolve to approximately equal scales. The adjustment of the current sheet thickness to the ion gyroradius scale leads to an electron-ion charge separation in the current sheet and, consequently, to a strong normal electric field at, and in back of the current sheet. We have shown that, in the frame of the moving current sheet, this electric field component can be understood as due to a large Hall contribution but also to an almost equally large, and opposing, electron pressure gradient contribution. A pileup region forms in front of the propagating current sheet in which the plasma density, pressure, temperature (not shown), flow speed, and $B_{z}$ field strength rise from undisturbed values found far in front of the current sheet to their respective disturbed values at the current sheet. A dawnward drifting region of both ions and electrons forms within the pileup region. Surprisingly, although the electrons and ions drift together they do not drift at the expected $\mathbf{E} \times \mathbf{B}$ speed. For the electrons, the difference has been shown to be due to an important thermal pressure gradient term in the momentum equation (7). We have found a similar explanation for the ion drifts (not shown). However, in the case of the ions, all of the terms in (7) play important roles and none can be neglected.

[65] We have found that the DF-1 simulation exhibits ion reflection and acceleration in the pileup region. A model for the motion of the ions in the dominant $B_{z}$ field of the pileup region has been used to estimate the energy gain in the acceleration process. Comparing the measured energy gain of the reflected gyrophase bunched ions in the DF-1 simulation to the prediction of the model has led to the conclusion that the ions are reflected by a normal electric field directed earthward at the leading edge of the pileup region in conjunction with the $B_{z}$ field there, not through an interaction with the current sheet and its associated magnetic field gradient. The reflection mechanism appears similar to that which produces the gyrophase bunched ions observed upstream of Earth's bow shock. We have found that the highest energy ions in the undisturbed upstream plasma gain a factor of $2.5-3.5$ times their initial energy through the reflection process.

[66] We have analyzed the contributions in the pileup region of the DF-1 simulation of the terms of the ion momentum equation (13) to understand the earthward acceleration of that region. We have found that the thermal pressure gradient dominates at the leading edge of the pileup region, the electromagnetic force density peaks up at a lower value just in back of the thermal peak, and deeper in the pileup region both the electromagnetic and thermal pressure gradient force densities are comparable and relatively weaker. Much of the earthward acceleration is provided by the thermal pressure gradient and electromagnetic force densities at the leading edge of the pileup region as plasma is gathered up there by the oncoming disturbance. It is not possible to reduce the electromagnetic term of (13) to MHD concepts generally but it is reasonable to think of a magnetic pressure gradient contribution at the leading edge of the pileup region where the field strength grows rapidly toward the interior of the region. To this degree, the DF-1 simulation results are consistent with those of Li et al. [2011] with an earthward total pressure gradient force density accelerating the pileup region in the simulation and with a reduction in the tailward total pressure gradient force density providing the acceleration in the magnetotail. More generally, without the assumption of a magnetic pressure gradient contribution to the electromagnetic term, it can be said that the DF-1 simulation results provide a somewhat generalized but compatible interpretation of the $\mathrm{Li}$ et al. results.

[67] We have found that wave activity at the leading edge of the pileup region is a persistent feature of our DF simulations, including the DF-1 simulation. Wave activity growth coincides with the maximum gradient in density, pressure, field strength, etc., at the leading edge. This is also the region where the ion velocity distribution is highly nongyrotropic due to the presence of the reflected ions. Both the steep gradients and the nongyrotropic velocity distributions are candidates for exciting the waves. However, we have not identified the wave mode(s) involved.

[68] The wave activity is characterized by oscillating filaments of current in the dawn-dusk direction. The wavelength in the north-south direction is approximately two ion inertial lengths, which is also comparable to the ion gyroradius in the pileup region. By tracking the position of maximum wave activity as it propagates with the leading edge of the pileup region, we have been able to determine that the waves oscillate at that position at approximately four times the ion cyclotron frequency.

[69] The pileup region is characterized by high electron and ion betas. The current filament waves are excited in high beta plasma.

[70] Perturbations in the electromagnetic field are associated with the current filament waves. In the magnetic field, the waves are largely restricted to the perpendicular (to $B_{z}$ ) components. The perpendicular magnetic field perturbations rotate about $B_{z}$ in a right-handed sense with layers separated by approximately one ion inertial length containing opposing perturbation vectors. The perturbation magnitudes are almost phase independent, leading to almost circularly polarized wave activity. The electric field component $E_{z}$ contains only the ideal frozen-in contribution, showing that the wave activity is stationary in the reference frame moving with the plasma in the earthward direction.

[71] We have shown that the strength of the magnetic field perturbations associated with the current filament waves is typically a few tenths of the strength of the $B_{z}$ field in the pileup region. By placing an observation point in the wavefield as the disturbance propagates by, we have found that the combination of fast earthward propagation speed, narrow wavefield disturbance, and oscillation period commensurate with the disturbance passage time leads to a short-lived unpredictable disturbance at any given observation point. Very small spatial displacements result in the appearance of unrelated signatures. No clear relationship between the evolutions of the perpendicular field components is discernible. 
[72] The presence of the current filament waves in plasma sheet DF observations may have gone unnoticed so far. Detection of the waves would be challenging.

[73] Acknowledgments. We thank Don Fairfield for many helpful discussions. This research was supported by NASA's MMS IDS grant NCC5-494 (MOST).

[74] Philippa Browning thanks the reviewers for their assistance in evaluating this paper.

\section{References}

Angelopoulos, V., C. F. Kennel, F. V. Coroniti, R. Pellat, M. G. Kivelson, R. J. Walker, C. T. Russell, W. Baumjohann, W. C. Feldman, and J. T. Gosling (1994), Statistical characteristics of Bursty bulk flow events, J. Geophys. Res., 99(A11), 21,257-21,280.

Angelopoulos, V., et al. (2008), First results from the THEMIS mission, Space Sci. Rev., 141(1-4), 453-476.

Büchner, J., and L. M. Zelenyi (1989), Regular and chaotic charged-particle motion in magnetotail-like field reversals. 1. Basic theory of trapped motion, J. Geophys. Res., 94(A9), 11,821-11,842.

Dubyagin, S., V. Sergeev, S. Apatenkov, V. Angelopoulos, R. Nakamura, J. McFadden, D. Larson, and J. Bonnell (2010), Pressure and entropy changes in the flow-braking region during magnetic field dipolarization, J. Geophys. Res., 115, A10225, doi:10.1029/2010JA015625.

Eastman, T. E., R. R. Anderson, L. A. Frank, and G. K. Parks (1981), Upstream particles observed in the earth's foreshock region, J. Geophys. Res., 86(NA6), 4379-4395.

Gurgiolo, C., G. K. Parks, B. H. Mauk, C. S. Lin, K. A. Anderson, R. P. Lin, and H. Reme (1981), Non-E X B ordered ion-beams upstream of the earth's bow shock, J. Geophys. Res., 86(NA6), 4415-4424.

Gurgiolo, C., G. K. Parks, and B. H. Mauk (1983), Upstream gyrophase bunched ions - A mechanism for creation at the bow shock and the growth of velocity space structure through gyrophase mixing, J. Geophys. Res., 88(NA11), 9093-9100.

Hesse, M., K. Schindler, J. Birn, and M. Kuznetsova (1999), The diffusion region in collisionless magnetic reconnection, Phys. Plasmas, 6(5), $1781-1795$.

Hwang, K. J., M. L. Goldstein, E. Lee, and J. S. Pickett (2011), Cluster observations of multiple dipolarization fronts, J. Geophys. Res., 116, A00i32, doi:10.1029/2010JA015742.

Klimas, A., M. Hesse, and S. Zenitani (2008), Particle-in-cell simulation of collisionless reconnection with open outflow boundaries, Phys. Plasmas, 15, 082102, doi:10.1063/1.2965826.

Klimas, A., M. Hesse, S. Zenitani, and M. Kuznetsova (2010), Particlein-cell simulation of collisionless driven reconnection with open boundaries, Phys. Plasmas, 17, 112,904, doi:10.1063/1.3510480.

Klimas, A., M. Hesse, and S. Zenitani (2012), Particle-in-cell simulation of collisionless undriven reconnection with open boundaries, Phys. Plasmas, 19, 042,901, doi:10.1063/1.3699032.

Langdon, A. B. (1992), On enforcing Gauss law in electromagnetic particlein-cell codes, Comput. Phys. Commun., 70(3), 447-450.

Leroy, M. M., C. C. Goodrich, D. Winske, C. S. Wu, and K. Papadopoulos (1981), Simulation of a perpendicular bow shock, Geophys. Res. Lett., $8(12), 1269$.

Li, S. S., V. Angelopoulos, A. Runov, X. Z. Zhou, J. McFadden, D. Larson, J. Bonnell, and U. Auster (2011), On the force balance around dipolarization fronts within bursty bulk flows, J. Geophys. Res., 116, A00i35, doi:10.1029/2010JA015884.

Mende, S. B., H. U. Frey, V. Angelopoulos, and Y. Nishimura (2011), Substorm triggering by poleward boundary intensification and related equatorward propagation, J. Geophys. Res., 116, A00i31, doi:10.1029/2010JA015733.

Nakamura, R., A. Retino, W. Baumjohann, M. Volwerk, N. Erkaev, B. Klecker, E. A. Lucek, I. Dandouras, M. Andre, and Y. Khotyaintsev (2009), Evolution of dipolarization in the near-Earth current sheet induced by Earthward rapid flux transport, Ann. Geophys., 27(4), 1743-1754.
Nishimura, Y., L. Lyons, S. Zou, V. Angelopoulos, and S. Mende (2010), Substorm triggering by new plasma intrusion: THEMIS all-sky imager observations, J. Geophys. Res., 115, A07222, doi:10.1029/2009JA015166.

Ohtani, S. (2004), Flow bursts in the plasma sheet and auroral substorm onset: Observational constraints on connection between midtail and near-Earth substorm processes, Space Sci. Rev., 113(1-2), 77-96.

Ohtani, S., Y. Miyashita, H. Singer, and T. Mukai (2009), Tailward flows with positive B-Z in the near-Earth plasma sheet, J. Geophys. Res., 114, A06218, doi:10.1029/2009JA014159.

Panov, E. V., et al. (2010a), Multiple overshoot and rebound of a bursty bulk flow, Geophys. Res. Lett., 37, L08103, doi:10.1029/2009GL041971.

Panov, E. V., et al. (2010b), Plasma sheet thickness during a bursty bulk flow reversal, J. Geophys. Res., 115, A05213, doi:10.1029/2009JA014743.

Pritchett, P. L. (2008), Collisionless magnetic reconnection in an asymmetric current sheet, J. Geophys. Res., 113, A06210, doi:10.1029/2007JA012930.

Pritchett, P. L., and J. Buchner (1995), Collisionless reconnection in configuration with a minimum in the equatorial magnetic-field and with magnetic shear, J. Geophys. Res., 100(A3), 3601-3611.

Runov, A., V. Angelopoulos, M. I. Sitnov, V. A. Sergeev, J. Bonnell, J. P. McFadden, D. Larson, K. H. Glassmeier, and U. Auster (2009), THEMIS observations of an earthward-propagating dipolarization front, Geophys. Res. Lett., 36, L14106, doi:10.1029/2009GL038980.

Runov, A., V. Angelopoulos, X. Z. Zhou, X. J. Zhang, S. Li, F. Plaschke, and J. Bonnell (2011a), A THEMIS multicase study of dipolarization fronts in the magnetotail plasma sheet, J. Geophys. Res., 116, A05216, doi:10.1029/2010JA016316.

Runov, A., et al. (2011b), Dipolarization fronts in the magnetotail plasma sheet, Planet. Space Sci., 59(7), 517-525.

Schmid, D., M. Volwerk, R. Nakamura, W. Baumjohann, and M. Heyn (2011), A statistical and event study of magnetotail dipolarization fronts, Ann. Geophys., 29(9), 1537-1547.

Sergeev, V. A., V. Angelopoulos, S. Apatenkov, J. Bonnell, R. Ergun, R. Nakamura, J. McFadden, D. Larson, and A. Runov (2009), Kinetic structure of the sharp injection/dipolarization front in the flow-braking region, Geophys. Res. Lett., 36, L21105, doi:10.1029/2009GL040658.

Sergeev, V. A., V. Angelopoulos, and R. Nakamura (2012), Recent advances in understanding substorm dynamics, Geophys. Res. Lett., 39, L05101, doi:10.1029/2012GL050859.

Shiokawa, K., W. Baumjohann, and G. Haerendel (1997), Braking of highspeed flows in the near-Earth tail, Geophys. Res. Lett., 24(10), 1179-1182.

Sibeck, D. G., and V. Angelopoulos (2008), THEMIS Science Objectives and Mission Phases, Space Sci. Rev., 141(1-4), 35-59.

Sitnov, M. I., and M. Swisdak (2011), Onset of collisionless magnetic reconnection in two-dimensional current sheets and formation of dipolarization fronts, J. Geophys. Res., 116, A12216, doi:10.1029/2011JA016920.

Sitnov, M. I., M. Swisdak, and A. V. Divin (2009), Dipolarization fronts as a signature of transient reconnection in the magnetotail, J. Geophys. Res., 114, A04202, doi:10.1029/2008JA013980.

Xing, X., L. R. Lyons, V. Angelopoulos, D. Larson, C. Carlson, A. Runov, and U. Auster (2010), Plasma sheet pressure evolution related to substorms, J. Geophys. Res., 115, A01212, doi:10.1029/2009JA014315.

Zhou, M., M. Ashour-Abdalla, X. H. Deng, D. Schriver, M. El-Alaoui, and Y. Pang (2009), THEMIS observation of multiple dipolarization fronts and associated wave characteristics in the near-Earth magnetotail, Geophys. Res. Lett., 36, L20107, doi:10.1029/2009GL040663.

Zhou, X. Z., V. Angelopoulos, V. A. Sergeev, and A. Runov (2010), Accelerated ions ahead of earthward propagating dipolarization fronts, J. Geophys. Res., 115, A00i03, doi:10.1029/2010JA015481.

Zhou, X. Z., V. Angelopoulos, V. A. Sergeev, and A. Runov (2011), On the nature of precursor flows upstream of advancing dipolarization fronts, J. Geophys. Res., 116, A03222, doi:10.1029/2010JA016165.

Zhou, X. Z., V. Angelopoulos, A. Runov, J. Liu, and Y. S. Ge (2012a), Emergence of the active magnetotail plasma sheet boundary from transient, localized ion acceleration, J. Geophys. Res., 117, A10216, doi:10.1029/2012JA018171.

Zhou, X. Z., Y. S. Ge, V. Angelopoulos, A. Runov, J. Liang, X. Xing, J. Raeder, and Q. G. Zong (2012b), Dipolarization fronts and associated auroral activities: 2 . Acceleration of ions and their subsequent behavior, J. Geophys. Res., 117, A10227, doi:10.1029/2012JA017677. 